\title{
Bootstrap Dynamical Symmetry Breaking
}

\author{
Wei-Shu Hou \\ Department of Physics, National Taiwan University, Taipei 10617, Taiwan \\ Correspondence should be addressed to Wei-Shu Hou; wshou@phys.ntu.edu.tw
}

Received 22 June 2012; Accepted 24 February 2013

Academic Editor: Tao Han

Copyright (c) 2013 Wei-Shu Hou. This is an open access article distributed under the Creative Commons Attribution License, which permits unrestricted use, distribution, and reproduction in any medium, provided the original work is properly cited.

\begin{abstract}
Despite the emergence of a $125 \mathrm{GeV}$ Higgs-like particle at the LHC, we explore the possibility of dynamical electroweak symmetry breaking by strong Yukawa coupling of very heavy new chiral quarks $Q$. Taking the $125 \mathrm{GeV}$ object to be a dilaton with suppressed couplings, we note that the Goldstone bosons $G$ exist as longitudinal modes $V_{L}$ of the weak bosons and would couple to $Q$ with Yukawa coupling $\lambda_{\mathrm{Q}}$. With $m_{\mathrm{Q}} \gtrsim 700 \mathrm{GeV}$ from LHC, the strong $\lambda_{\mathrm{Q}} \gtrsim 4$ could lead to deeply bound $Q \bar{Q}$ states. We postulate that the leading "collapsed state," the color-singlet (heavy) isotriplet, pseudoscalar $Q \bar{Q}$ meson $\pi_{1}$, is $G$ itself, and a gap equation without Higgs is constructed. Dynamical symmetry breaking is affected via strong $\lambda_{\mathrm{Q}}$, generating $m_{\mathrm{Q}}$ while self-consistently justifying treating $G$ as massless in the loop, hence, "bootstrap," Solving such a gap equation, we find that $m_{Q}$ should be several TeV, or $\lambda_{Q} \gtrsim 4 \pi$, and would become much heavier if there is a light Higgs boson. For such heavy chiral quarks, we find analogy with the $\pi$ - $N$ system, by which we conjecture the possible annihilation phenomena of $Q \bar{Q} \rightarrow n V_{L}$ with high multiplicity, the search of which might be aided by Yukawa-bound $Q \bar{Q}$ resonances.
\end{abstract}

\section{Introduction and Motivation}

The field of particle physics is in a state of both jubilation and anxiety. On one hand, the long-awaited Higgs boson seems to have finally emerged $[1,2]$ at $\sim 125 \mathrm{GeV}$ at the LHC, where a hint appeared already with 2011 data $[3,4]$. The case has been further strengthened with more data added by end of 2012, and the final results for the $7-8 \mathrm{TeV}$ run would be revealed by the Moriond meetings in 2013. On the other hand, there appears to be no New Physics below the $\mathrm{TeV}$ scale, and one is worried what really stabilizes the Higgs mass at $125 \mathrm{GeV}$.

As much as the existence of a $125 \mathrm{GeV}$ boson is beyond doubt, we note that it is not yet experimentally established that it is the Standard Model (SM) Higgs boson. In part because of the enhancement in $\gamma \gamma$ mode-for both ATLAS and CMS, and for both 7 and $8 \mathrm{TeV}$ data-and also because the subdominant production channels are not yet experimentally firm, the $125 \mathrm{GeV}$ object could still be a dilaton [5-7]. The dilaton couples like a Higgs boson, but with couplings suppressed by $v / f$, where $v$ is the observed vacuum expectation value (v.e.v.) of electroweak symmetry breaking (EWSB), and $f$ is the "dilaton decay constant" that is related to scale-invariance violation. The dilaton couplings to $g g$ and $\gamma \gamma$, however, are determined by the trace anomaly of the energy-momentum tensor and would depend on UV details. Keeping $v / f$ and effective $g g$ and $\gamma \gamma$ couplings free, it is found [6] that a "dilaton" interpretation is as consistent as an SM-Higgs. To reject the dilaton, one has to establish the subdominant vector boson fusion (VBF) and Higgsstrahlung, or associated production $(\mathrm{VH})$ processes at the expected SM level. Judging from the results available at the end of 2012, it seems [8] that the issue would have to await the restart of the $\mathrm{LHC}$ at $13 \mathrm{TeV}$.

Regardless of whether the $125 \mathrm{GeV}$ object is the SM Higgs boson or a dilaton, the Higgs mechanism is an experimental fact. That is, the electroweak (EW) gauge symmetry is experimentally established, while the gauge bosons, as well as the chirally charged fermions, are all found to be massive, in apparent violation of the gauge symmetry. Thus, the Goldstone particle of EWSB gets "eaten" by the EW gauge bosons, which become massive (the Meissner effect), as has been experimentally established since 30 years. The v.e.v. $v$ is simply related to the venerable Fermi constant $G_{F}$.

With both quarks and gauge bosons massive, a heuristic argument was used to demonstrate [9] that, starting from the left-handed vector gauge coupling, the longitudinal 


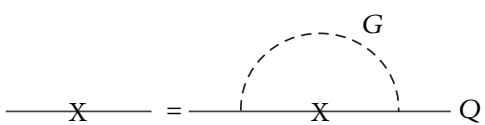

FIGURE 1: Gap equation for generating heavy quark mass from Goldstone boson loop.

component of the EW gauge boson, that is, equivalently the Goldstone boson $G$, couples to quarks by the SM Yukawa coupling, with both left- and right-handed components. Thus, Yukawa couplings are experimentally established. Furthermore [9], much of flavor physics and $C P$ violation (CPV) studies probe the effects of Yukawa couplings, providing ample and highly nontrivial support for their "complex" existence.

A natural question to ask is as follows: given three quark generations already, could there be a fourth copy? Does it carry its own raison d'être? It is not our purpose to discuss in detail the issues, merits, and demerits regarding this possible fourth generation $(4 \mathrm{G})$, which we refer to [10]. At face value, we admit that the observed $125 \mathrm{GeV}$ new boson would pose a difficulty. The main issue is not so much the existence of the Higgs boson, but one as light as $125 \mathrm{GeV}$. The gluongluon fusion production, through the top loop in SM, is now augmented by $4 \mathrm{G}$ quarks $t^{\prime}$ and $b^{\prime}$ in the loop, leading to an enhancement of order $(1+1+1)^{2}=9$ in production cross-section, which does not seem reflected by data. In fact, searches [11] assuming this enhancement factor rule out a Higgs boson in the full mass range up to $600 \mathrm{GeV}$. We are reminded, however, that by simply extending from $3 \rightarrow 4$ generations, the effective CPV increases [12] by a thousand-trillion-fold or more and may provide enough CPV to satisfy the Sakharov conditions for baryogenesis. Given that the three-generation Kobayashi-Maskawa model [13] falls far short of the needed CPV, Nature might use such an enhancement factor. Furthermore, before one rules out the dilaton possibility, the premise for the order of magnitude enhancement in production may not stand.

Fourth generation $t^{\prime}$ and $b^{\prime}$ quarks have been pursued vigorously at the LHC, as it should be done for a hadron collider, independent of the Higgs situation. The current bound $[14-20]$ is

$$
m_{\mathrm{Q}} \gtrsim 700 \mathrm{GeV} \text {, }
$$

where $Q$ stands for a chiral quark doublet, in which the limit is from both $t^{\prime}$ and $b^{\prime}$ search. We shall assume "heavy isospin" symmetry, $I_{Q}$, and treat the doublet $Q$ as degenerate, which can be viewed as part of the custodial SU(2) symmetry. What is important is that the current $m_{\mathrm{Q}}$ bound is already above the nominal perturbative partial wave unitarity bound (UB) of $550 \mathrm{GeV}$ [21]. The Yukawa coupling $\lambda_{\mathrm{Q}} \equiv \sqrt{2} m_{\mathrm{Q}} / v \gtrsim 4 \lambda_{t}$ (where $\lambda_{t} \simeq 1$ ) has already entered the strong coupling regime; $\alpha_{\mathrm{Q}} \equiv \lambda_{\mathrm{Q}}^{2} / 4 \pi \gtrsim 1$.

With the ever increasing bound on $m_{Q}$, the fourth generation may well not exist. But being beyond UB, a new question is as follows: could the strong Yukawa coupling of $Q$ generate [22] EWSB itself? This is an intriguing conjecture and provides a second reason for having a fourth generation. Along this line, a gap equation, given symbolically in Figure 1, was constructed [9] without ever invoking the Higgs doublet, that is, the Higgs boson field of SM.

The logic or philosophy went as follows. The Goldstone boson $G$ is viewed as a tightly bound $Q \bar{Q}$ state, bound by the Yukawa coupling itself. Guided by a Bethe-Salpeter equation study [23], strong Yukawa binding could lead to state collapse; that is, the bound state turns tachyonic, which is taken as suggestive of triggering EWSB itself. For further elucidation, see [24]. Reference [9] went one step further to postulate that the leading collapsed state, the color-singlet isosinglet pseudoscalar $Q \bar{Q}$ meson, $\pi_{1}$, is the Goldstone boson $G$ itself. With no New Physics in sight at the LHC, not even the heavy chiral quark $Q$ itself, the loop momentum integration runs up to roughly $2 m_{\mathrm{Q}}$ (when the Goldstone boson ceases to exist), without the need to add any further effects (in the ladder approximation of truncating corrections to $G$ propagation and GQQ vertex). This is therefore a "bootstrap" gap equation, in that the strong Yukawa coupling itself is the source of EWSB, or mass generation for quark $Q$, which simultaneously justifies keeping the Goldstone $G$ in the loop (the $125 \mathrm{GeV}$ object is assumed to be the dilaton). The existence of a large Yukawa coupling $\lambda_{Q}$ is used as input, without a theory for $\lambda_{\mathrm{Q}}$ itself. There is no attempt at UV completion.

It is important then to investigate whether one could find a solution to such a gap equation. If so, we would have demonstrated the case for dynamical symmetry breaking (DSB) and the potential riches that could follow. This paper offers a comprehensive elucidation of the line of thought from the aforementioned, through the demonstration of DSB, and linking the need of $2-3 \mathrm{TeV}$ quarks to the possible new phenomenon of $Q \bar{Q} \rightarrow n V_{L}$ production, the search of which could be aided by Yukawa resonances.

In Section 2, we trace the arguments that set up the bootstrap gap equation, including the postulate of the Goldstone boson $G$ as a "collapsed state." In Section 3, we formulate this gap equation more clearly, and demonstrate that numerical solution does exist [8]. A similar gap equation was formulated by Hung and Xiong [25], where $G$ in Figure 1 is replaced by a massless Higgs doublet field. We will compare and offer a critique. Our numerical solution [8] suggests $m_{Q}$ to be in the $2-3 \mathrm{TeV}$ range, corresponding to $\lambda_{\mathrm{Q}} \gtrsim 4 \pi$. Even for $m_{\mathrm{Q}}$ lower than this range, one may ask whether the usual assumption of $p p \rightarrow Q \bar{Q}+X$ followed by free $Q$ decay would hold, as assumed so far in direct searches [1420]. The large GQQ Yukawa coupling now resembles the $\pi N N$ coupling in strength; to our surprise, we find $g_{\pi N N} \simeq$ $\lambda_{\pi N N} \equiv \sqrt{2} m_{N} / f_{\pi} \sim 4 \pi$. We tap into the known $p \bar{p} \rightarrow$ $n \pi$ phenomena and conjecture that $Q \bar{Q} \rightarrow n V_{L}$, with multiplicity $\sim 6-12$, and with a characteristic temperature of order $v$ that is to be measured. This is discussed in Section 4 . We end with further discussions and offer a conclusion in Section 5 .

\section{A Gap Equation without Higgs}

My own interest in the 4 th generation was revitalized by a hint of possible New Physics in electroweak penguin 
contributions to direct CPV in $B \rightarrow K \pi$ decay (e.g., see, the account given in [12]). In between 2007 and 2008, my interest turned to direct search for $b^{\prime}$ and $t^{\prime}$ at the LHC with the CMS experiment. Although initially I wished for $m_{b^{\prime}} \lesssim 300 \mathrm{GeV}$ for sake of the rich phenomenology [26, 27], I found the link [22] of strong Yukawa coupling with EWSB rather intriguing. As the direct search limits on $t^{\prime}$ and $b^{\prime}$ rose, I began to find the usual Higgs mechanism via an elementary Higgs doublet more and more problematic. Having just $\mu^{2}<0$ in the Lagrangian lacked dynamics (it is only a description), while Nature seems to be saying something through the absence of elementary scalars in QED and QCD, where the dynamics are better understood than EWSB. Furthermore, most problems such as the hierarchy problem arose by treating the Higgs doublet field as elementary.

The following is how curiosity led the way from a flavor/CPV entry into dynamical EWSB, without invoking the existence of an elementary Higgs doublet field.

2.1. Yukawa Coupling from Gauge Coupling. Holding the SMHiggs interpretation of the $125 \mathrm{GeV}$ particle observed $[1,2]$ at LHC as still suspect, let us recall the firm facts from experiment.

First, we know [28] that all observed quarks $q$ and charged leptons $l$ are pointlike to smaller than $10^{-18} \mathrm{~m}$, and they are governed by the $\mathrm{SU}(3)_{\mathrm{C}} \otimes \mathrm{SU}(2)_{\mathrm{L}} \otimes \mathrm{U}(1)_{\mathrm{Y}}$ gauge dynamics. Chromodynamics would not be our concern, but it is important to emphasize that, unlike the 1970s and early 1980s, the $\mathrm{SU}(2)_{\mathrm{L}} \otimes \mathrm{U}(1)_{\mathrm{Y}}$ chiral gauge dynamics is now experimentally established. We know that quarks and leptons come in lefthanded weak doublets and right-handed singlets, and for each given electric charge, they carry different hypercharge $Y$.

Second, the weak bosons are found [28] to be massive; $M_{W}=(1 / 2) g v$, where $g$ is the measured $\mathrm{SU}(2)_{\mathrm{L}}$ weak coupling, and $v^{2}=1 / \sqrt{2} G_{F}$ the vacuum expectation value. Hence, spontaneous breaking of $\mathrm{SU}(2)_{\mathrm{L}} \otimes \mathrm{U}(1)_{\mathrm{Y}}$ symmetry (SSB) is also experimentally established.

Third, all fermions are observed [28] to be massive. These masses also manifest EWSB, since they link leftand right-handed fermions of the same electric charge, but different $\mathrm{SU}(2)_{\mathrm{L}}$ and $\mathrm{U}(1)_{\mathrm{Y}}$ charges. We shall not invoke the elementary Higgs boson for mass generation, as this is the question we explore, and because the dilaton possibility has to be ruled out by experiment.

At this point, we need to acknowledge the important theoretical achievement of renormalizability [29] of nonAbelian gauge theories, which allowed theory-experiment correspondence down to per mille level precision, especially for the extensive work [28] done at the LEP, SLC, as well as TeVatron colliders. The proof of renormalizability is based on Ward identities, hence, [30] unaffected by SSB; that is, the underlying symmetry properties are not affected. From this, we now demonstrate $[9,31]$ the existence of Yukawa couplings as experimental fact.

With proof of renormalizability, we choose the physical unitary gauge; hence, there are no would-be Goldstone bosons (or unphysical scalars), only massive gauge bosons

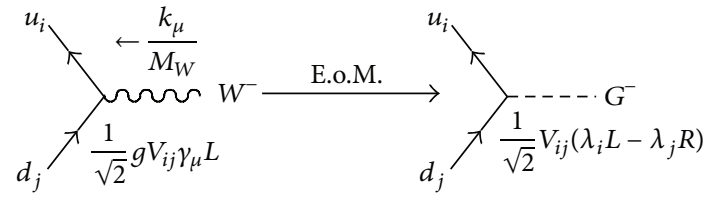

FIGURE 2: Deriving the Yukawa coupling, that is, Goldstone boson coupling to quarks, from purely left-handed gauge couplings, where E.o.M. stands for equation of motion.

(strictly speaking, only the on-shell $S$-matrix is finite in $U$ gauge, we thank T. Kugo for the comment and take it as a limiting case of the $R_{\xi}$ gauge for sake of illustration). Longitudinal $W$ boson (the would-be Goldstone bosons that got "eaten") propagation is via the $\left(\left(k_{\mu} k_{v}\right) / M_{W}^{2}\right)$ part of the $W$ boson propagator. If we take a $k_{\mu} / M_{W}$ factor and contract with a $d_{j} \rightarrow u_{i}$ charged current, as illustrated in Figure 2, simple manipulations give (dropping $V_{i j}$ for convenience)

$$
\begin{aligned}
\frac{g}{\sqrt{2}} \frac{k}{M_{W}} L & =\frac{g}{\sqrt{2}} \frac{\not p_{i}-\not p_{j}}{M_{W}} L \\
& =\frac{g}{\sqrt{2}} \frac{m_{i} L-m_{j} R}{M_{W}} \\
& =\sqrt{2}\left(\frac{m_{i}}{v} L-\frac{m_{j} R}{v}\right) \\
& \equiv \lambda_{i} L-\lambda_{j} R,
\end{aligned}
$$

where we have inferred

$$
\lambda_{\mathrm{Q}} \equiv \frac{\sqrt{2} m_{\mathrm{Q}}}{v}
$$

as the effective $W_{L}$, or Goldstone boson $G$ coupling to quarks, which is nothing but the familiar Yukawa coupling. We have used the equation of motion, that is, the Dirac equation, on $d_{j}$ and $u_{i}$ quarks in the second step of (2), but this is justified since we exist in the broken phase for the real world, and we know experimentally that all quarks are massive.

From Figure 2 and equations (2) and (3), we see that from the experimentally well-established left-handed gauge coupling, the Goldstone boson couples via the usual Yukawa coupling. The Goldstone bosons of EWSB pair with the transverse gauge boson modes to constitute a massive gauge boson, the Meissner effect, but the important point is that we have not introduced a physical Higgs boson in any step. Unlike the Higgs boson, SSB of electroweak symmetry is an experimentally established fact. The Goldstone bosons couple with Yukawa couplings proportional to fermion mass, independent of whether it arises from an elementary Higgs doublet.

We have kept a factor $V_{i j}$ in Figure 2. Recall that the Kobayashi-Maskawa (KM) formalism [13] for quark mixing deals with massive quarks, or equivalently the existence of Yukawa matrices, and the argument remains exactly the same. Vast amount of flavor and CP violation (CPV) data overwhelmingly supports [28] the 3 generation KM picture. 


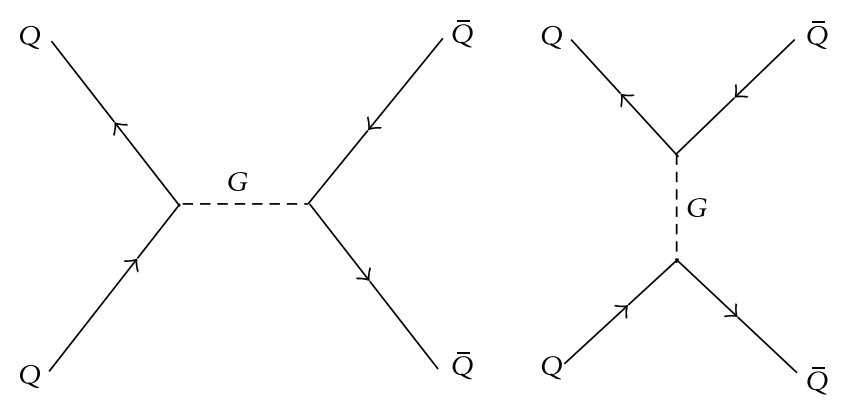

FIGURE 3: Scattering diagrams for $t$ - and $s$-channel Goldstone boson $G$ exchange between $Q$ and $\bar{Q}$ quarks. Analogous diagrams can be drawn for $g$ (and $H$ ) exchange.

For example, the unique CPV phase with 3 quark generations can so far explain all observed CPV phenomena. These facts further attest to the existence of Yukawa couplings from their dynamical effects but again do not provide any evidence for the existence of the SM Higgs boson.

2.2. A Postulate: Collapse of Yukawa Bound State. Based on experimental facts and the renormalizability of electroweak theory, we have "derived" Yukawa couplings from purely left-handed gauge couplings in the previous section, without invoking an explicit Higgs sector, at least not at the empirical, heuristic level. We turn now to a more hypothetical situation: could there be more chiral generations? Since we already have three, the possibility that there exists a fourth generation of quarks should not be dropped in a cavalier way. We note again that increasing to 4 generations, one may have sufficient amount of CPV for baryogenesis [12] from the KM mechanism. There has also been some resurgent recent interest $[10,14-20]$. As argued in the Introduction, although the spirit may have been dampened by the $125 \mathrm{GeV}$ Higgs-like object, one should press on with the direct search.

What we do know is that the $t^{\prime}$ and $b^{\prime}$ quarks should be suitably degenerate to satisfy electroweak constraints on the $S$ and $T$ variables [32]. A "heavy isospin" is in accordance with the custodial SU(2) symmetry.

2.2.1. Relativistic Expansion versus Bethe-Salpeter Equation. With $m_{t^{\prime}} \cong m_{b^{\prime}} \equiv m_{\mathrm{Q}} \gtrsim 700 \mathrm{GeV}$ [14-20], $\lambda_{\mathrm{Q}}$ is already 4 times stronger than the top quark, hence, stronger than all gauge couplings. There have been two complementary studies of strong Yukawa bound states. The first approach is along traditional lines of relativistic expansion [33]. Ignoring all gauge couplings except QCD, and taking the heavy isospin limit, with $Q$ representing the 4 th generation quark doublet, and $G$ the triplet of Goldstone bosons, the $t$ - and $s$-channel Goldstone exchange diagrams are depicted in Figure 3, with corresponding diagrams for $g$ as well as $H$ exchange ([33] did not put in $s$-channel gluon exchange).

The heavy $\bar{Q} Q$ mesons form isosinglets and isotriplets and can be color singlet or octet. We borrow the notation from hadrons and call these states $\eta_{1}, \omega_{1}, \pi_{1}, \rho_{1}$, and $\eta_{8}, \omega_{8}, \pi_{8}$, $\rho_{8}$, respectively. Reference [33] used a variational approach, with radius $a_{0}$ as parameter. It was found that, for color singlet $\omega_{1}\left(\rho_{1}\right), a_{0} / a_{\mathrm{QCD}} \sim 1$ for $m_{\mathrm{Q}}$ below $400(540) \mathrm{GeV}$, but above which $a_{0}$ suddenly precipitates towards tiny values. For $\eta_{1}\left(\pi_{1}\right)$, the radius mildly decreases (increases) from 1 , with trend reversed for binding energy; hence, it remains QCDbound.

To understand this, note that the $t$-channel Goldstone exchange for $\eta_{1}$ is repulsive, while the $s$-channel Goldstone exchange, contributing only to $\pi_{1}$, is also repulsive. However, the sudden drop in $\omega_{1}$ and $\rho_{1}$ radii is due to the trial wave function sensing suddenly a lower energy at tiny radius due to $t$-channel Goldstone exchange; the strong Yukawa coupling has wrested control of binding from the Coulombic QCD potential. QCD binding energy is only a couple of $\mathrm{GeV}$, but the sudden drop in radius leads to a sharp rise in binding energy, hence, a kink. One finds that the relativistic $v / c$ expansion fails just when it starts to get interesting. For color octet states, QCD is repulsive; so, $\eta_{8}$ does not bind. In [33], the $\omega_{8}$ and $\pi_{8}$ states are degenerate, with sudden shrinking of radius occurring around $530 \mathrm{GeV}$, but the $s$-channel QCD effect, left out in [33], should push the $\omega_{8}$ upwards; the $\rho_{8}$ state does not shrink until later.

Given that the relativistic expansion breaks down, a truly relativistic approach is needed. Such a study, based on a Bethe-Salpeter (BS) equation [23], was pursued around the time of demise of the SSC, for very heavy chiral quark doublets. The BS equation is a ladder sum of $t$ - and $s$ channel diagrams of Figure 3, where the $\bar{Q} Q$ pair forms a heavy meson bound state. While the ladder sum of $t$ channel diagrams is intuitive, a problem emerges for the $s$ channel, which contributes only to $\pi_{1}, \omega_{8}$, and $\sigma_{1}$ (same quantum numbers as $G, g$, and $H$, resp.). Rather than a triangle loop, the $s$-channel loop appears like a self-energy, hence, potentially divergent, while the momentum carried by the exchanged boson is the bound state mass itself. One could not formally turn the integral equation into an eigenvalue problem. This was resolved in [23] by a subtraction at fixed external momentum, which in effect eliminates all $s$ channel diagrams. Reference [23] then solved the BS equation numerically using several different approximations, which, in addition to the approximate nature of the BS equation itself, illustrates the uncertainties. Still, unlike [33], the bound state masses drop smoothly below $2 m_{Q}$ as $m_{Q}$ increases, showing no kink, which is an improvement from a relativistic expansion. However, a generic feature is collapse; bound state masses tend to drop sharply to zero at some high $m_{\mathrm{Q}}$ and would naively turn tachyonic.

2.2.2. Postulate for Leading Collapsed State. Here, we do not pursue the more conservative phenomenology for precollapse Yukawa couplings as in [24] but wish to address more fundamental issues. Although the de facto $s$-channel subtraction made by [23] appeared reasonable on formal grounds, the contrast with the relativistic expansion is striking; the Goldstone $G$ exchange in $s$-channel led to a specific repulsion [33] for $\pi_{1}$ heavy mesons, disallowing it to shrink suddenly like the otherwise analogous $\omega_{1}$. But after subtracting the $s$-channel, the $\pi_{1}$ becomes [23] the most attractive channel (MAC), more so than $\omega_{1}$. Together with the tendency towards 
collapse for large enough $m_{\mathrm{Q}}$ (equivalently $\lambda_{\mathrm{Q}}$ ), this means that the $\pi_{1}$ meson would be the first to drop to zero and turn tachyonic. That this occurs for the channel that experiences repulsion when $2 m_{Q}$ is far lower than collapse values (à la $\omega_{1}$ which has no $s$-channel effect) seems paradoxical. Does this falsify the whole approach, or else what light does this shed? And how is it related to $s$-channel subtraction?

With experimental bounds [14-20] for 4th generation quarks entering the region of deep(er) binding, we offer a self-consistent view that may seem radical. Clearly, around and below $500 \mathrm{GeV}$ mass, or $2 m_{\mathrm{Q}} \lesssim 1 \mathrm{TeV}$, there could still be some repulsion due to $G$ exchange in $s$-channel. But since we did not introduce any elementary Higgs doublet, the Goldstone boson $G$ should perhaps be viewed as a $\bar{Q} Q$ bound state. Hence, we

Postulate $\left(\pi_{1} \equiv G\right)$. Collapse is a precursor to dynamical EWSB, and the first mode to collapse becomes the Goldstone mode.

Although the full validity of the BS equation may be questioned, it is known [34] that "the appearance of a tachyonic bound state leads to instability of the vacuum," which is "resolved by condensation into the tachyonic mode." Our postulate removes the equation for $\pi_{1}$ self-consistently (there is another aspect on the self-consistency of this subtraction/postulate, if one did not remove the $\pi_{1}$ equation by invoking this subtraction, the leading collapse state of strong Yukawa coupling would be the $\omega_{1}$, condensation in this channel would break Lorentz invariance) and provides some understanding of the $s$-channel subtraction; a $\pi_{1} / G$ boson carrying $p^{2} \sim\left(2 m_{\mathrm{Q}}\right)^{2}$ would no longer be a bound Goldstone boson in $s$-channel. Without an elementary Higgs boson, there is no $\sigma_{1}$ channel subtraction, while for heavy enough $m_{\mathrm{Q}}$ (so $\pi_{1}$ has turned Goldstone), one can treat QCD effects as a correction, after solving the $\omega_{8}$ bound state problem, without need of subtracting $s$-channel $g$ exchange. The selfconsistent MAC behavior of the $\pi_{1}$ channel seems like a reasonable outcome of the Goldstone dynamics, as implied by the gauge dynamics.

It may now appear that EWSB is some kind of a "bootstrap" from "massive chiral quarks" with large Yukawa coupling as seen in broken phase.

2.3. A Bootstrap Gap Equation without Higgs. Motivated by the previous heuristic discussion, we construct a gap equation for the dynamical generation of heavy quark mass without invoking the Higgs boson.

For a long time, as $m_{\mathrm{Q}}$ limit rose, I focused on breaching the UB, which the experimental pursuit would not be concerned about. Although UB violation (UBV) occurs at far higher energy, once the experimental limits breach the bound, some form of strong interactions would take over. I asked myself how UBV might be amended by Nature. The study of Yukawa bound states [24] arose from this pursuit, but did not shed sufficient light on the issue, except approaching the abyss of state "collapse", as described earlier.
A mindset change occurred when one connected two of the $Q$ or $\bar{Q}$ lines in Figure 3. One gets the self-energy for $Q$ by $G$ exchange and readily arrives at the gap equation [9] as depicted in Figure 1. If quark mass $m_{Q}$, represented by the cross $\mathrm{X}$, could be nonzero, then one has dynamical chiral symmetry breaking, which is equivalent to EWSB! Both $Q$ and $G$ are treated as massless at the diagrammatic level, since the gap equation is summing over all possible momenta $q$ carried by $G$ as it mediates-dominates-Q $\bar{Q} \rightarrow Q \bar{Q}$ scattering. But if $|q|$ reaches $2 m_{Q}$, there would no longer be a Goldstone boson $G$ (assuming $G$ is a $Q \bar{Q}$ bound state), or it would be resolved in the $t$-channel. Thus, the summation over $q^{2}$ should not exceed $\left(2 m_{\mathrm{Q}}\right)^{2}$ at the heuristic level. The whole picture is heuristic, but realistic in the experimental sense, since the gap equation integrates over a large momentum range of $q^{2}$, where we have no other New Physics that enter, as indicated by LHC data.

Such a gap equation was constructed recently from a different, and in our view more ad hoc, theoretical argument. In [25], an elementary Higgs doublet is assumed together with a 4th generation. Motivated by their earlier study [35, 36], where some UV fixed point (UVFP) behavior was conjectured, these authors pursued dynamical EWSB via a Schwinger-Dyson equation that is rather similar to our Figure 1. However, perhaps in anticipation of the UVFP that might develop at high energy $[35,36]$, they put in by hand a massless Higgs doublet, hence, a scale invariant theory to boot. It is the massless Higgs doublet that runs in the loop, replacing our Goldstone boson $G$. The massless nature of the Higgs doublet appears ad hoc, and the paper defers the discussion of the physical Higgs spectrum for a future work.

In contrast, our Goldstone boson $G$, identified as the collapsed $\pi_{1}$ state as it turns tachyonic, is strictly massless in the broken phase. In the gap equation of Figure 1, we speculate that the loop momentum should be cut off around $2 m_{\mathrm{Q}}$, rather than some "cut-off" scale $\Lambda>2 m_{\mathrm{Q}}$. In so doing, we bypass all issues of triviality that arise from having $\Lambda$ approaching $2 m_{\mathrm{Q}}$. What happens at scales above $2 m_{\mathrm{Q}}$ is to be studied by experiment.

Here, we remark that the first, elementary Higgs of [25], $\pi$ and $\sigma$ are our bound state Goldstone bosons, and indeed we should have a $\sigma$-like massive broad bound state [24] that could mimic the heavy Higgs boson. Their second Higgs doublet, in the form of $t^{\prime}$ and $b^{\prime}$ bound states, would be excitations above the $\pi_{1}$ and $\sigma_{1}$ for us, likely rather broad. We think that their claimed third doublet, that of bound $\tau^{\prime}$ and $v_{\tau}^{\prime}$, may not be bound at all, as their Yukawa couplings may not be large enough.

The gap equation illustrated in Figure 1 actually links to a vast literature on strongly coupled, scale-invariant QED. It is known that such a theory could have spontaneous chiral symmetry breaking when couplings are strong enough. We turn to our numerical study [8] in the next section, where we also offer our critique of [25].

Although our line of thought may seem constructed, we have developed a self-consistent picture where EWSB from large Yukawa coupling may be realized with some confidence-all without assuming an elementary Higgs 


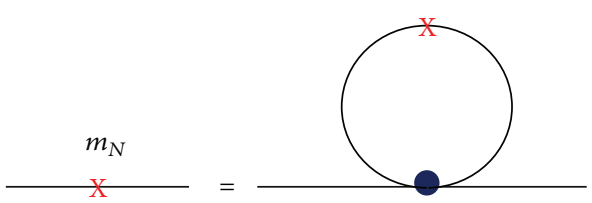

Figure 4: The gap equation for the Nambu-Jona-Lasinio model for generating nucleon mass.

boson. We have not yet really touched on the $\sigma_{1}$ meson, which would be the heavy Higgs boson. However, our scenario is to have Goldstone bosons as strongly (and tightly) bound "Cooper pairs" of very heavy quarks, which may please Nambu. For the $125 \mathrm{GeV}$ Higgs-like object, the inherent scaleinvariant nature of our gap equation allows the possibility of a dilaton interpretation, as we will discuss later.

\section{Solving Bootstrap Gap Equation}

The question now is whether the symbolic gap equation of Figure 1 affords actual solutions. Towards finding a solution, in this section, we briefly review the Nambu-Jona-Lasinio model, where one sets up a gap equation with its well-known solution. We then turn to the so-called strongly coupled scale-invariant QED, which is closer to our gap equation. By recounting some major steps, we also set up our notation for later usage. We find a coupled set of integral equations, which is more complicated than strong QED in Landau gauge. But a numerical solution is found [8], with $\lambda_{Q} \gtrsim 4 \pi$, where we compare and contrast with [25].

3.1. NJL Model: p-Independent Self-Energy. The NambuJona-Lasinio model [37] is the earliest, explicit model of DSB, where the breaking of global chiral symmetry leads to generation of nucleon mass, and the pion as a (pseudo-) Goldstone boson [38-40]. (Strictly speaking, the Goldstone boson should really be called the Nambu-Goldstone (NG) boson, since [38] predated [39]. But for sake of notation, and because of more common usage, we shall use $G$ and Goldstone boson throughout our paper).

The model is depicted in Figure 4, where a four-fermion interaction is introduced (the blob on the right-hand side). The nucleon mass, represented by a cross, is self-consistently generated. One easily finds the gap equation

$$
\begin{aligned}
m_{N} & =\frac{N_{C}}{8 \pi^{2}} G \int_{0}^{\Lambda^{2}} d q^{2} q^{2} \frac{m_{N}}{q^{2}+m_{N}^{2}} \\
& =\frac{N_{C}}{8 \pi^{2}} G \Lambda^{2}\left(1-\frac{m_{N}^{2}}{\Lambda^{2}} \log \left(1+\frac{\Lambda^{2}}{m_{N}^{2}}\right)\right) m_{N}
\end{aligned}
$$

where $G$ here is the four-fermi coupling and $\Lambda$ is the cutoff. Since $m_{N}$ on both sides factor out, one has

$$
1-\frac{G_{\text {crit }}}{G}=\frac{m_{N}^{2}}{\Lambda^{2}} \log \left(1+\frac{\Lambda^{2}}{m_{N}^{2}}\right)
$$

which admits a solution for $G>G_{\text {crit }}$, with

$$
G_{\text {crit }}=\frac{8 \pi^{2}}{N_{C} \Lambda^{2}}
$$

To understand what is happening, one can iterate the cross of the left-hand side of Figure 4 on the right-hand side and see that it constitutes an infinite number of diagrams. This effectively puts the original self-energy diagram into the denominator. In the end, one trades the parameters $G$ and $\Lambda$ for the physical $m_{N}$ and the pion-nucleon coupling. At the more refined level and using the quark language, one can show further that the emergent Goldstone boson, the pion, is in fact a ladder sum of the quark-level four-fermi interaction.

We will return at the end to discuss the similarity and differences of the NJL model with our gap equation.

3.2. Strong QED: p-Dependent Self-Energy. We note that the self-energy bubble of Figure 4 does not depend on external momentum $p$, and so at the superficial level, Figure 4 is quite different from Figure 1. We now turn to QED, where there is much closer similarity.

The general gap equation for QED [41] can be written in the form of the Schwinger-Dyson (SD) equation

$$
\Sigma(p)=i e^{2} \int \frac{d^{4} q}{(2 \pi)^{4}} \gamma^{\mu} D_{\mu \nu}(q) S(p-q) \Gamma^{\nu}(p, q)
$$

where $\Sigma=\not p-m-S(p)^{-1}$ is the electron self-energy with $S$ the (full) electron propagator, $D_{\mu \nu}$ is the (full) photon propagator, and $\Gamma$ is the full vertex.

3.2.1. Ladder Approximation. Truncating the exact, full vertex and photon propagator by the approximation

$$
\begin{gathered}
\Gamma^{\nu}(p, q)=\gamma^{\nu}, \\
D_{\mu \nu}(q)=\frac{-g_{\mu \nu}+q^{\mu} q^{\nu} / q^{2}}{q^{2}}-\xi \frac{q_{\mu} q_{\nu}}{q^{4}}
\end{gathered}
$$

called the ladder (or rainbow) approximation, but retaining the electron self-energy, the gap equation becomes

$$
S(p)^{-1}=\not p-i e^{2} \int \frac{d^{4} q}{(2 \pi)^{4}} \gamma^{\mu} D_{\mu \nu}(p-q) S(q) \gamma^{\nu}
$$

with $D_{\mu \nu}$ now given in (9), and we have set $m=0$, that is, massless QED at Lagrangian level. Pictorially, this is represented as in Figure 5, where we note that, compared with the four-fermion coupling of the NJL model depicted in Figure 4, the external momentum $p$ now flows into the selfenergy loop. The question now is whether chiral symmetry can be dynamically broken, that a nontrivial solution to the self-energy could be generated at some strong coupling $\alpha=$ $e^{2} / 4 \pi$ ? 


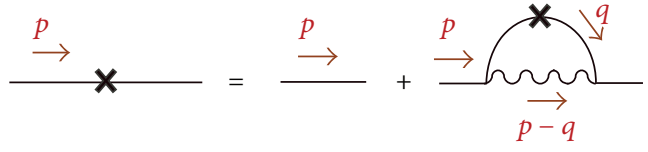

FIGURE 5: Gap equation for QED in the ladder approximation.

We define the electron propagator as [41]

$$
S(p)=\frac{1}{A\left(p^{2}\right) \not p-B\left(p^{2}\right)},
$$

where the $A$ term corresponds to wave function (w.f.) renormalization related to the usual $Z^{-1}$ factor. A finite pole of the propagator, $p^{2}=B^{2}\left(p^{2}\right) / A^{2}\left(p^{2}\right)$, would give the dynamical effective mass. Our aim is therefore solving $A$ and $B$ from the gap equation of (10). Inserting (9) and after some algebra, one finds

$$
\begin{gathered}
B\left(p^{2}\right)=e^{2} \int \frac{i d^{4} q}{(2 \pi)^{4}}\left(\frac{-4+1-\xi}{k^{2}}\right) \frac{B\left(q^{2}\right)}{A^{2}\left(q^{2}\right) q^{2}-B^{2}\left(q^{2}\right)}, \\
A\left(p^{2}\right)=1-\frac{e^{2}}{4 p^{2}} \operatorname{tr} \int \frac{i d^{4} q}{(2 \pi)^{4}} \gamma^{\mu} D_{\mu \nu}^{(\xi)} \frac{A\left(q^{2}\right) \not d}{A^{2}\left(q^{2}\right) q^{2}-B^{2}\left(q^{2}\right)} \gamma^{\nu} \not p .
\end{gathered}
$$

Simplification can be achieved in the Landau gauge, $\xi=0$, where one finds $A\left(p^{2}\right)=1$. Since $A$ relates to w.f. renormalization, it satisfies the Ward-Takahashi identity even under the ladder approximation. The gap equation is simplified to a single equation

$$
B\left(p^{2}\right)=-3 e^{2} \int \frac{i d^{4} q}{(2 \pi)^{4}} \frac{B\left(q^{2}\right)}{(p-q)^{2}\left(q^{2}-B^{2}\left(q^{2}\right)\right)} .
$$

After Wick rotation, and using

$$
\begin{aligned}
& \int d^{4} q \frac{f\left(q^{2}\right)}{(p-q)^{2}} \\
& =\pi^{2} \int d q^{2} q^{2}\left[\frac{\theta(p-q)}{p^{2}}+\frac{\theta(q-p)}{q^{2}}\right] f\left(q^{2}\right),
\end{aligned}
$$

one obtains

$$
B(x)=\frac{3 \alpha}{4 \pi}\left(\frac{1}{x} \int_{\Lambda_{\mathrm{IR}}^{2}}^{x} d y \frac{y B(y)}{y+B^{2}(y)}+\int_{x}^{\Lambda^{2}} d y \frac{B(y)}{y+B^{2}(y)}\right),
$$

where $x=p_{E}^{2}$ is the Euclidean momentum squared, and $\Lambda^{2}, \Lambda_{\text {IR }}^{2}$ are the ultraviolet (UV) and infrared (IR) cutoffs, respectively.

The integral equation can be changed to a differential equation by noting

$$
\begin{gathered}
\frac{d B(x)}{d x}=\frac{3 \alpha}{4 \pi}\left(-\frac{1}{x^{2}}\right) \int_{\Lambda_{\mathrm{IR}}^{2}}^{x} d y \frac{y B(y)}{y+B^{2}(y)} \\
\frac{d(x B(x))}{d x}=\frac{3 \alpha}{4 \pi} \int_{x}^{\Lambda^{2}} d y \frac{B(y)}{y+B^{2}(y)} .
\end{gathered}
$$

One obtains the differential equation

$$
x \frac{d^{2} B(x)}{d x^{2}}+2 \frac{d B(x)}{d x}+\frac{3 \alpha}{4 \pi} \frac{B(x)}{x+B^{2}(x)}=0
$$

together with the IR and UV boundary conditions (B.C.)

$$
\left.\frac{d B(x)}{d x}\right|_{x=\Lambda_{\mathrm{IR}}^{2}}=0,\left.\quad \frac{d(x B(x))}{d x}\right|_{x=\Lambda^{2}}=0 .
$$

If IR cutoff is 0 , the B.C. for IR should be replaced by $2 B d B / d x=-3 \alpha / 4 \pi$ at $x=0$.

3.2.2. Solution in Landau Gauge. The coupled integral equations are simplified and put into a differential equation with B.C. In order to study qualitative features, let us first find an approximate solution. For a special range for the IR cutoff, let us take $B(x)=\Lambda_{\text {IR }}<x^{1 / 2}<\Lambda$; then, (17) is simplified to

$$
x^{2} \frac{d^{2} B(x)}{d x^{2}}+2 x \frac{d B(x)}{d x}+\frac{3 \alpha}{4 \pi} B(x)=0 .
$$

Inserting $B(x)=C x^{\lambda}$, the characteristic equation is

$$
\lambda(\lambda-1)+2 \lambda+\frac{3 \alpha}{4 \pi}=0
$$

with discriminant $1-3 \alpha / \pi$; hence, the behavior is different for $\alpha>\alpha_{c}$ and $\alpha<\alpha_{c}$, where $\alpha_{c}=\pi / 3$. The analytical solution under the approximation is given as

$$
\begin{aligned}
B & =b_{1} x^{\left(-1+\sqrt{1-\alpha / \alpha_{c}}\right) / 2}+b_{2} x^{\left(-1-\sqrt{1-\alpha / \alpha_{c}}\right) / 2} \\
& \equiv b_{1} x^{c_{1}}+b_{2} x^{c_{2}}
\end{aligned}
$$

and the boundary conditions can be written as

$$
\left(\begin{array}{cc}
c_{2} & c_{1} \Lambda_{\mathrm{IR}}^{2\left(c_{1}-c_{2}\right)} \\
-1 & \Lambda^{2\left(c_{1}-c_{2}\right)}
\end{array}\right)\left(\begin{array}{l}
b_{1} \\
b_{2}
\end{array}\right)=\left(\begin{array}{l}
0 \\
0
\end{array}\right)
$$

The determinant must vanish for nontrivial $b_{1}, b_{2}$; hence,

$$
\frac{\sqrt{1-\alpha / \alpha_{c}}-1}{\sqrt{1-\alpha / \alpha_{c}}+1}=\left(\frac{\Lambda^{2}}{\Lambda_{\mathrm{IR}}^{2}}\right)^{\sqrt{1-\alpha / \alpha_{c}}}
$$

To satisfy this condition, $\alpha>\alpha_{c}=\pi / 3$ is needed, and for given $\Lambda / \Lambda_{\text {IR }}, \alpha$ takes on discontinuous values

$$
n \pi+\tan ^{-1} \frac{1}{\sqrt{\alpha / \alpha_{c}-1}}=\sqrt{\alpha / \alpha_{c}-1} \log \frac{\Lambda}{\Lambda_{\mathrm{IR}}} .
$$

One sees that $\alpha \rightarrow \alpha_{c}$ for $\Lambda / \Lambda_{\text {IR }} \rightarrow \infty$, the nominal "continuum limit." For $\alpha<\alpha_{c}$, the only solution that satisfies the B.C. is the trivial $B(x)=0$. 


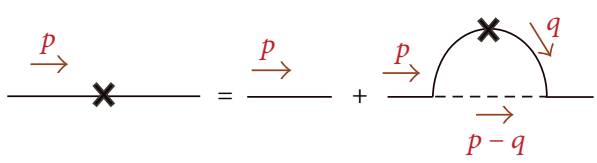

Figure 6: Gap equation for large Yukawa coupling in the ladder approximation, where vanishing $m_{0}$ is indicated.

The approximate solution for small IR cutoff can now be obtained by replacing $B(x)=m$ (constant) for small $x$. The differential equation becomes

$$
x \frac{d^{2} B(x)}{d x^{2}}+2 \frac{d B(x)}{d x}+\frac{3 \alpha}{4 \pi} \frac{B(x)}{x+m^{2}}=0,
$$

where $B(x)$ is a hypergeometric function. Namely,

$$
B(x)=m F\left(\frac{1}{2}-i \gamma, \frac{1}{2}+i \gamma, 2 ;-\frac{x}{m^{2}}\right)
$$

where

$$
i \gamma=\frac{1}{2} \sqrt{1-\frac{\alpha}{\alpha_{c}}}, \quad \alpha_{c}=\frac{\pi}{3},
$$

and $F$ is a hypergeometric function. Checking the asymptotic behavior for $x \gg m^{2}$, the power behavior for $\alpha<\alpha_{c}$ cannot satisfy the UV boundary condition.

The gap equation has a nontrivial or oscillatory solution for $\alpha>\alpha_{c}$, where the critical coupling is $\alpha_{c}=\pi / 3$ for QED. The dynamical effective mass is obtained by solving $x=B^{2}(-x)$. Note that the pole of the propagator should be given in the time-like region; so, one has to make analytical continuation in order to obtain a physical mass, which can be done smoothly for $\Lambda_{\text {IR }} \rightarrow 0$.

An issue arises in that the dynamical mass

$$
m=4 \Lambda e^{-\pi / \sqrt{\alpha / \alpha_{c}-1}}
$$

is proportional to the UV cutoff $\Lambda$. For $m$ to be physical, however, it should not depend on $\Lambda$. Miransky suggested [42] that $\alpha \rightarrow \alpha_{c}$ as $\Lambda \rightarrow \infty$; that is, $\alpha_{c}$ is a nontrivial UV fixed point. A related issue, which we would not go into, is whether there would be a dilaton associated with breaking of scale invariance $[43,44]$. (These two are also the two best references for gauged NJL model).

3.3. Bootstrap Gap Equation for EWSB. Our recapitulation of strong QED is for the purpose of setting up our approach for solving the bootstrap gap equation [9] with large empirical Yukawa coupling, where we will continue to follow the Fukuda-Kugo approach.

In Landau gauge $(\xi=0)$, the propagations of (would-be) Goldstone modes and gauge bosons are properly separated, and the gap equation becomes equivalent to our discussion if one takes the $g \rightarrow 0$ limit for the gauge coupling. The Goldstone bosons $G^{ \pm}$and $G^{0}$ couple to fermions with the familiar Yukawa couplings, which we have argued [9] as experimentally established. They are also unaltered by the $g \rightarrow 0$ limit, as seen in (2). The main assumption is the addition of a new (heavy) chiral quark doublet $Q$, where the heavy isospin $I_{Q}$ symmetry implies that $\lambda_{U}=\lambda_{D} \equiv \lambda_{Q}$, that is, equality of the $I_{Q 3}= \pm 1 / 2$ Yukawa couplings. We ask whether large $\lambda_{\mathrm{Q}}$ could be the source of EWSB, through the conceptual gap equation of Figure 1 .

3.3.1. Ladder Approximation. In any case, we do not know the full propagator and vertex functions. We approximate the Goldstone-fermion vertex as undressed, and the Goldstone propagator remains as $i / k^{2}$, analogous to the QED treatment in previous section. This is in part aided by the insight that $G$ is a very tight $Q \bar{Q}$ bound state, which would be massless as long as the symmetry remains spontaneously broken. Similar to Figure 5, the gap equation for large Yukawa (vanishing $g$ ) coupling is depicted in Figure 6. Again, the external momentum $p$ flows through the loop.

The change from the symbolic or conceptual equation of Figure 1 is that, even with bare mass forbidden by gauge invariance $(m=0)$, there is a $\not p$ part for $Q$ propagation, and we aim at solving for the quark propagator $S(p)$, given as

$$
S(p)^{-1}=A\left(p^{2}\right) \not p-B\left(p^{2}\right)
$$

which is of course of the same form as (11) for QED. The Goldstone boson $G$ is colorless, and, unlike the massless photon from electromagnetic gauge invariance for QED case, its masslessness is "bootstrapped" into the gap equation itself. Following similar steps as in Section 2, and assuming $\lambda_{U}=$ $\lambda_{D}=\lambda_{\mathrm{Q}}$ degeneracy, we obtain

$$
\begin{aligned}
B_{p^{2}}= & \frac{3 \lambda_{Q}^{2}}{2} \int \frac{d^{4} q}{i(2 \pi)^{4}} \frac{1}{(p-q)^{2}} \frac{B_{q^{2}}}{q^{2} A_{q^{2}}^{2}-B_{q^{2}}^{2}} \\
& -\frac{\lambda_{Q}^{2}}{2} \int \frac{d^{4} q}{i(2 \pi)^{4}} \frac{1}{(p-q)^{2}-m_{h}^{2}} \frac{B_{q^{2}}}{q^{2} A_{q^{2}}^{2}-B_{q^{2}}^{2}}, \\
A_{p^{2}=} & +\frac{3 \lambda_{Q}^{2}}{2 p^{2}} \int \frac{d^{4} q}{i(2 \pi)^{4}} \frac{p \cdot q}{(p-q)^{2}} \frac{A_{q^{2}}}{q^{2} A_{q^{2}}^{2}-B_{q^{2}}^{2}} \\
& +\frac{\lambda_{Q}^{2}}{2 p^{2}} \int \frac{d^{4} q}{i(2 \pi)^{4}} \frac{p \cdot q}{(p-q)^{2}-m_{h}^{2}} \frac{A_{q^{2}}}{q^{2} A_{q^{2}}^{2}-B_{q^{2}}^{2}}
\end{aligned}
$$

where placement of $i$ anticipates the Wick rotation, and $B_{q^{2}}$ stands as shorthand for $B\left(q^{2}\right)$, and likewise for $A$. We have already used $\xi=0$; so, compared to massless QED, we now have to consider $A$, or wave function renormalization effects; hence, we have to face a coupled set of equations for $A\left(p^{2}\right)$ and $B\left(p^{2}\right)$. Note that we have kept a "Higgs" term for calculational purposes, applying Standard Model Higgs boson, $h^{0}$, couplings. This is for purpose of later comparison with the work of Hung and Xiong [25], as well as for discussion of the dilaton case.

For our aim of bootstrap DSB, we simply drop the second term (no physical Higgs, or taking $m_{h} \sim \infty$ ), and so only 
Goldstone modes propagate in both equations. After angular integration and Wick rotation, one gets

$$
\begin{array}{r}
B(x)=\kappa_{b}\left(\frac{1}{x} \int_{\Lambda_{\mathrm{IR}}^{2}}^{x} d y \frac{y B(y)}{y A^{2}(y)+B^{2}(y)}\right. \\
\left.+\int_{x}^{\Lambda^{2}} d y \frac{B(y)}{y A^{2}(y)+B^{2}(y)}\right), \\
A(x)=1+\kappa_{a}\left(\frac{1}{x^{2}} \int_{\Lambda_{\mathrm{IR}}^{2}}^{x} d y \frac{y^{2} A(y)}{y A^{2}(y)+B^{2}(y)}\right. \\
\left.+\int_{x}^{\Lambda^{2}} d y \frac{A(y)}{y A^{2}(y)+B^{2}(y)}\right),
\end{array}
$$

where

$$
\kappa_{b}=2 \kappa_{a}=\frac{(3 / 2) \lambda_{Q}^{2}}{16 \pi^{2}}=\frac{(3 / 2) \alpha_{Q}}{4 \pi} \quad \text { (no Higgs). }
$$

Taking the limit $m_{h} \rightarrow 0$ in (30) and (31) to mimic the HungXiong approach of massless scalar doublet,

$$
\kappa_{b}=\kappa_{a}=\frac{\lambda_{\mathrm{Q}}^{2}}{16 \pi^{2}}=\frac{\alpha_{\mathrm{Q}}}{4 \pi} \quad \text { (massless Higgs). }
$$

We keep the notation of $\kappa_{b}$ and $\kappa_{a}$ in (33) and (34) to cover these two cases.

Analogous to Section 2, (32) can be put into differential form

$$
\begin{aligned}
& x B^{\prime \prime}+2 B^{\prime}+\frac{\kappa_{b} B}{x A^{2}+B^{2}}=0, \\
& x A^{\prime \prime}+3 A^{\prime}+\frac{2 \kappa_{a} A}{x A^{2}+B^{2}}=0,
\end{aligned}
$$

with the boundary conditions

$$
\begin{gathered}
\left.B^{\prime}(x)\right|_{x=\Lambda_{\mathrm{IR}}^{2}}=0, \\
{\left.\left[x B^{\prime}(x)+B(x)\right]\right|_{x=\Lambda^{2}}=0,} \\
\left.A^{\prime}(x)\right|_{x=\Lambda_{\mathrm{IR}}^{2}}=0, \\
{\left.\left[\frac{x}{2} A^{\prime}(x)+A(x)\right]\right|_{x=\Lambda^{2}}=1,}
\end{gathered}
$$

where prime stands for $x$-derivative.

At this point, we note that, if one ignores wave function renormalization, that is, (36), while forcefully setting $A(x)=$ 1 in (35), then one has the same solution as in QED, with the change in critical coupling

$$
\alpha_{\mathrm{Q}}^{c}=\frac{2 \pi}{3}
$$

which is twice as high as for QED, and

$$
\lambda_{\mathrm{Q}}^{c}=2 \pi \sqrt{\frac{2}{3}} \simeq 5.13 ;
$$

hence, superficially a "critical mass" $m_{Q}^{c} \simeq 890 \mathrm{GeV}$, which is above current LHC limits [14-20]. But it should be clear that the wave function renormalization $A\left(p^{2}\right)$ term cannot be neglected.
3.3.2. Numerical Solution. Redefining $p^{2}=x=e^{2 t}$, our coupled differential equations with B.C. become

$$
\begin{gathered}
\ddot{B}+2 \dot{B}+\frac{4 \kappa_{b} B}{A^{2}+B^{2} e^{-2 t}}=0, \\
\ddot{A}+4 \dot{A}+\frac{8 \kappa_{a} A}{A^{2}+B^{2} e^{-2 t}}=0, \\
\dot{B}\left(t_{\mathrm{IR}}\right)=0, \quad \dot{B}\left(t_{\mathrm{UV}}\right)+B\left(t_{\mathrm{UV}}\right)=0, \\
\dot{A}\left(t_{\mathrm{IR}}\right)=0, \quad \frac{1}{4} \dot{A}\left(t_{\mathrm{UV}}\right)+A\left(t_{\mathrm{UV}}\right)=1,
\end{gathered}
$$

where dot represents $t$-derivative, and $e^{t_{\mathrm{UV}}}=\Lambda_{\mathrm{UV}}=\Lambda, e^{t_{\mathrm{IR}}}=$ $\Lambda_{\text {IR }}$.

Asymptotic Properties and Critical Coupling. Due to scale invariance, the differential equations are invariant under

$$
\begin{gathered}
x \longrightarrow a^{2} x \quad(t \longrightarrow t+\log a), \\
\Lambda_{\mathrm{UV}, \mathrm{IR}} \longrightarrow a \Lambda_{\mathrm{UV}, \mathrm{IR}}, \\
B \longrightarrow a B, \quad A \longrightarrow A .
\end{gathered}
$$

As a result, the solutions of the differential equations depend only on $\Lambda_{\mathrm{UV}} / \Lambda_{\mathrm{IR}}\left(=e^{t_{\mathrm{UV}}-t_{\mathrm{IR}}}\right)$ and $m_{\mathrm{dyn}} \equiv B\left(t_{\mathrm{IR}}\right) / A\left(t_{\mathrm{IR}}\right)$ for given $\kappa_{a}$ and $\kappa_{b}$. Thus, $m_{\mathrm{dyn}}$ is a kind of integration constant. We will see that the most important feature of the solutions is that only special values (discontinuous values) of $\kappa_{a}$ and $\kappa_{b}$ are allowed for given boundary conditions.

If we take $\kappa_{a}=0$, the equations can be solved analytically, and the solution can be described as in the case of strong coupling QED with Landau gauge, (21)-(23). The property illustrated for $\kappa_{a}=0$ should hold also for $\kappa_{a} \neq 0$, where, depending on $\Lambda_{\mathrm{UV}} / \Lambda_{\mathrm{IR}}, \kappa_{a}$ should take on special discontinuous values to satisfy the gap equation for the cases of $\kappa_{a} / \kappa_{b}=1$ or $1 / 2$.

To see this, we note that the $B^{2} e^{-2 t}$ term in the denominators is irrelevant. This is because for $t \ll \log m$, the $t$ dependence of $A$ and $B$ is negligible due to the boundary conditions $\dot{A}\left(t_{\mathrm{IR}}\right)=\dot{B}\left(t_{\mathrm{IR}}\right)=0$. Therefore, to understand the behavior of the solution, one can take $t_{\mathrm{IR}} \sim \log m$ and consider the differential equation with $B^{2} e^{-2 t}$ dropped as follows:

$$
\begin{aligned}
& \ddot{B}+2 \dot{B}+\frac{4 \kappa_{b} B}{A^{2}}=0, \\
& \ddot{A}+4 \dot{A}+\frac{8 \kappa_{a}}{A}=0,
\end{aligned}
$$

where the equation for $A$ becomes independent from $B$, but its solution affects $B$. 
The solution of $A$ is obtained analytically as follows:

$$
\begin{gathered}
A(x)=A_{0} \frac{\Lambda_{\mathrm{UV}}^{4}}{x^{2}} e^{-\zeta^{2}} \sqrt{\frac{\kappa_{a}}{\pi}} \\
\zeta=\operatorname{Erf}^{-1}\left[\frac{1}{A_{0}}\left(1-\frac{x^{2}}{\Lambda_{\mathrm{UV}}^{4}}\right)+\operatorname{Erf}\left(\frac{1}{\sqrt{\kappa_{a}}}\right)\right],
\end{gathered}
$$

where $A_{0}$ is an integration constant which can be fixed by the B.C. for IR. Using the analytical solution, one can show that only discontinuous values of $\kappa_{a, b}$ can satisfy the B.Cs. The "critical" value of $\kappa_{b}$ can be easily obtained numerically, even without neglecting $B^{2} e^{-2 t}$ term in the denominators. Of course, since the solutions are found numerically, it is not a proof that one really has a "critical" value. The upshot is that only special values of the coupling can satisfy the coupled equations for given values of $\Lambda_{\mathrm{UV}} / \Lambda_{\mathrm{IR}}$, for $m_{\mathrm{dyn}}<\Lambda_{\mathrm{IR}}$ (or $\Lambda_{\mathrm{UV}} / m_{\mathrm{dyn}}$ for $\left.m_{\mathrm{dyn}}>\Lambda_{\mathrm{IR}}\right)$ and $m_{\mathrm{dyn}}=B\left(\Lambda_{\mathrm{IR}}\right) / A\left(\Lambda_{\mathrm{IR}}\right)$.

Our numerical solution gives

$$
\begin{aligned}
& \kappa_{b}^{c} \simeq 1.4, \quad\left(\kappa_{b}=2 \kappa_{a}=\frac{3 \alpha_{Q}}{8 \pi}\right), \\
& \kappa_{b}^{c} \simeq 13.7, \quad\left(\kappa_{b}=\kappa_{a}=\frac{\alpha_{Q}}{4 \pi}\right),
\end{aligned}
$$

corresponding to

$$
\begin{aligned}
& \lambda_{\mathrm{Q}}^{c} \simeq 12, \quad\left(\kappa_{b}=2 \kappa_{a}=\frac{3 \alpha_{\mathrm{Q}}}{8 \pi}\right), \\
& \lambda_{\mathrm{Q}}^{c} \simeq 46, \quad\left(\kappa_{b}=\kappa_{a}=\frac{\alpha_{\mathrm{Q}}}{4 \pi}\right),
\end{aligned}
$$

where the latter case is much higher. Here $c$ stands for "critical," and our numerical values are extracted in the large $\Lambda_{\mathrm{UV}} / \Lambda_{\mathrm{IR}}$ and $\Lambda_{\mathrm{UV}} / m_{\mathrm{dyn}}$ limit. Note that for the artificial case of $\kappa_{a}=0$ (i.e., $A=1$ ), the critical value was $\lambda_{\mathrm{Q}}^{c} \simeq 5.1$, (40); hence, the effect of considering $A$ or wave function renormalization is quite nontrivial.

The values in (46) and (47) correspond to effectively taking $\Lambda_{\mathrm{UV}} \rightarrow \infty$, which is certainly not the range of validity for (46) as a descendent of Figure 1. That is, the conceptual foundation for Figure 1 is that, for momentum roughly up to somewhere below $2 m_{Q}$, corrections to the Goldstone boson propagator and vertex have been ignored. Nevertheless, at face value, if we naively apply the physical $v \simeq 246 \mathrm{GeV}$, then (46) and (47) imply the mass values

$$
m_{\mathrm{Q}}^{c}>2.1 \mathrm{TeV} \quad \text { (no Higgs) }
$$

and $8.1 \mathrm{TeV}$, respectively, which are rather high. We drop the latter, "massless (or light) Higgs" case, not only because it is clearly out of reach for the LHC, but for theoretical reasons as we explain later. The lower bound nature of (48) would be explained subsequently.

3.4. Discussion. The large critical coupling of $\lambda_{Q} \gtrsim 4 \pi$ of (46) is somewhat surprising, resulting in the rather high critical mass of (48). We note that this is without the contribution of a light SM Higgs boson, which would put $m_{\mathrm{Q}}^{c}$ much higher. Before we touch phenomenology of such high chiral quark masses, and in so doing find an independent justification for such high critical Yukawa coupling, we offer some further discussion.

3.4.1. Comparison with Hung and Xiong. We can now make some comparison with, and offer a critique of, the approach of Hung and Xiong [25] (HX). We have mimicked the concept of HX by keeping a "Higgs" scalar contribution in (30) and (31), which resulted in the second case of (34) in the limit of $m_{h} \rightarrow 0$, as compared with our case of interest, (33), where we drop the $m_{h}$-dependent term.

HX assumed the existence of a massless Higgs doublet, where our (35) and (36) with (34) should be a faithful representation. However, not only is the massless doublet assumed, HX also ignored wave function renormalization, that is, the $A$ term, in the treatment of their gap equation. Furthermore, the sign of our second integral in (30) disagrees with HX, suppressing the coefficient of $\lambda_{Q}^{2}$ in comparison. In the earlier work [9] that set up the gap equation of Figure 1, taking the numerics of $\mathrm{HX}$, the estimate of $\lambda_{\mathrm{Q}}^{c}=\sqrt{2} \pi$ (compare (40)) gave $m_{Q}^{c} \simeq 770 \mathrm{GeV}$, which is not too far above the current LHC bound. But with our sign for the second integral in (30), we would arrive at $\lambda_{Q}^{c}=2 \pi$, or $m_{\mathrm{Q}}^{c} \sim 1.1 \mathrm{TeV}$, that is, a factor of $\sqrt{2}$ higher. With our sign, the Higgs boson effect would cancel out part of the Goldstone effect, hence, requiring stronger Yukawa coupling.

But we have argued that it is not justified to ignore the wave function renormalization effect of $A\left(p^{2}\right)$. After all, the boson loop has momentum dependence, and so it would necessarily affect the $Z^{-1}$ factor. Thus, the previous simple numerics are incorrect. Keeping $A$ in our numerical study, hence, the coupled $A-B$ equations, a considerably higher critical $m_{\mathrm{Q}}^{c}$ is found. For the case of taking $\kappa_{b}=\kappa_{a}=$ $\lambda_{\mathrm{Q}}^{2} / 16 \pi^{2},(34)$, where we mimic HX's massless Higgs doublet effect, the critical $\lambda_{\mathrm{Q}}^{c}$ of (47) is almost 4 times as high as that for (46). In fact, we obtain the $A\left(\Lambda_{\mathrm{UV}}^{2}\right)=2.7$, which is quite different from 1 .

Our criticism goes far deeper. Taking a scalar doublet as massless, such that superficially one has "scale-invariance" as in strong QED, is totally ad hoc. Effectively one has to hold the parameters of the Higgs potential such that the Higgs field always remains massless. However, there is no principle by which this scale-invariance or masslessness of the Higgs field can be maintained. After all, one is invoking large Yukawa couplings, which feed the notorious divergent quadratic corrections to the Higgs boson mass. The one-loop two-point and four-point functions with quark $Q$ in the loop would generate effective $\mu^{2}$ and $\lambda$ self-coupling terms for the Higgs field. With no explicit dynamical principle (such as gauge invariance for the case of QED), the assumption of a massless Higgs doublet as the agent of DSB is not only ad hoc, but clearly unsustainable.

In contrast, we see the merit, as well as the meaning, of our "bootstrap" gap equation. As long as we are in the broken phase, there is a massless Goldstone boson that couples with 
the Yukawa coupling $\lambda_{\mathrm{Q}}$. Treating $\lambda_{\mathrm{Q}}$ as large, if a nontrivial solution to the gap equation is found (as we have illustrated earlier in the previous subsection), it in turn justifies the use of a massless Goldstone boson in the gap equation, which is why we affix the name "bootstrap." In fact, the physical argument [9] was to view the Goldstone boson $G$ as an extremely tight ultrarelativistic bound state of heavy $Q$ and $\bar{Q}$ from the broken phase, while $G$ enters the "bootstrap" gap equation to dynamically generate $m_{Q}$, hence, break the symmetry, and in the same stroke justify its own existence.

We also see that this argument for our bootstrap gap equation puts the existence of a light Higgs boson in doubt. That is, it seems rather difficult to keep it light, and one cannot ignore corrections to the Higgs propagator. At the foundation level [9], unlike the Goldstone boson, the experimental basis for the Higgs boson is rather recent. Even with the newly discovered [1,2] $125 \mathrm{GeV}$ boson, one has to distinguish between an SM Higgs boson versus a dilaton. Our numerical study also shows that keeping the Higgs term tends to raise the critical Yukawa coupling considerably, implying $2 m_{Q}$ higher than the LHC collision energy. Thus, the light Higgs case of $\kappa_{b}=\kappa_{a}$ is rather problematic in bootstrap DSB. We will return to discuss the dilaton possibility later.

3.4.2. Physical Cutoff. The previous comparison with [25], and in particular the bootstrap nature of the Goldstone boson $G$ in the gap equation, illustrates that the limit $\Lambda_{\mathrm{UV}} \rightarrow \infty$ would take us outside the range of validity of the gap equation itself. It is clear that, for timelike $q^{2}>\left(2 m_{\mathrm{Q}}\right)^{2}$, there would no longer be a Goldstone boson. Thus, for the cutoff $\Lambda$, one should not use the traditional language of $\Lambda_{\mathrm{UV}}$. One probably should not contemplate "UV-completion" at the current stage. The bootstrap gap equation is rather heuristically or physically argued. But it does not provide a theory of the heavy quark Yukawa coupling $\lambda_{\mathrm{Q}}$, employing it for DSB instead. Thus, we suggest a cutoff $\Lambda<2 m_{\mathrm{Q}}<\Lambda_{\mathrm{NP}}$, where $\Lambda_{\mathrm{NP}}$ is some true New Physics scale where the origin of Yukawa couplings may be contemplated, but it is out of reach from the bootstrap DSB approach.

Viewed from a slightly different angle, this cutoff is related to the restoration of symmetry. The Goldstone boson couples to broken currents, but the $Z$ factor of the Goldstone boson may vanish at some scale related to the scale where the Goldstone boson becomes unbounded, and symmetry is restored. Rather than a true $\Lambda_{\mathrm{UV}}$ that in principle extends to infinity, there exists a cutoff $\Lambda$ of the gap equation. We are in effect summing over the Goldstone boson correction to the self-energy of the quark $Q$, when the Goldstone boson is still defined. As noted in [9], this picture does receive experimental support, in that no New Physics seem to be there below $\sim \mathrm{TeV}$ scale. So, one sums up only the effect of the Goldstone boson, and nothing else.

The gap equation sums up the effect, from low to high momentum, of the correction by the Goldstone boson $G$ to the quark self-energy. Equation (46) reflects taking this sum to $\Lambda_{\mathrm{UV}} \rightarrow \infty$. Since the summation is accumulative, if now one sums only to some $\Lambda \leqslant 2 m_{\mathrm{Q}}$, as we have argued, then the cumulative effect is less than summing to very large

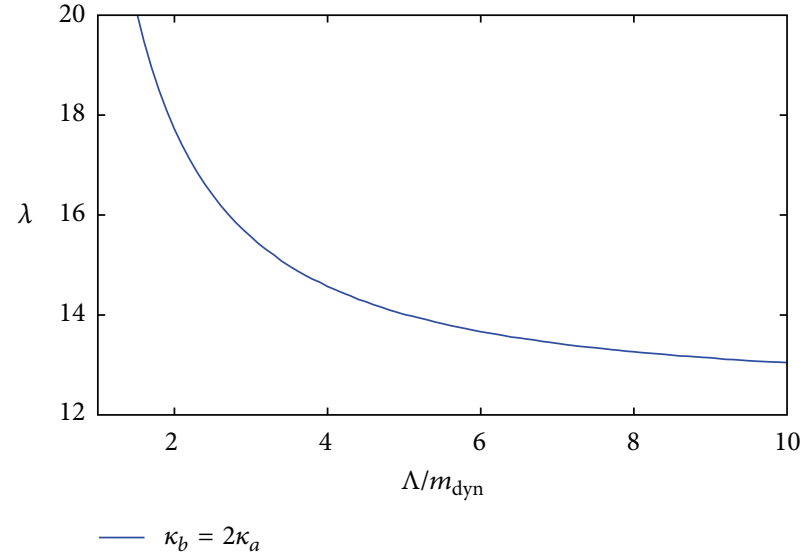

FIGURE 7: Cutoff dependence of Yukawa coupling, where the cutoff $\Lambda$ is in units of the dynamical quark mass $m_{\mathrm{dyn}}$.

momentum above $2 m_{\mathrm{Q}}$. We note that $2 m_{\mathrm{Q}}$ is a physical scale parameter that is external to the scale-invariant gap equation. Nevertheless, we can plot the dependence of $\lambda_{\mathrm{Q}}^{c}$ on the cutoff $\Lambda$. From Figure 7, one can see that, for a lower cutoff, $\lambda_{\mathrm{Q}}^{c}$ has to be higher than in (46). This is because, as the integration range is smaller, a larger $\lambda_{Q}$ value is needed to compensate. Thus, (46) gives a lower bound on $m_{\mathrm{Q}}$. If we take $\Lambda=2 m_{\mathrm{Q}}$, then $\lambda_{\mathrm{Q}}^{c} \sim 17.7$, and

$$
m_{\mathrm{Q}} \sim 3 \mathrm{TeV} \quad\left(\text { no Higgs; } \Lambda=2 m_{\mathrm{Q}}\right)
$$

which is higher than (48). We will return to discuss whether this could be an overestimate later.

3.4.3. Future Work. Up to now, we have been cavalier in the relation between $\lambda_{\mathrm{Q}}^{c}$ and $m_{\mathrm{Q}}^{(c)}$, treating it as $\sqrt{2} m_{\mathrm{Q}}^{(c)}=\lambda_{\mathrm{Q}}^{c} \nu$. But $v$ is a physically measured quantity, and $m_{Q}$ is yet to be experimentally measured. If electroweak symmetry is indeed dynamically broken by the large Yukawa coupling of a new heavy chiral quark $Q$, when $Q$ is discovered in the future, likely $m_{\mathrm{Q}}>m_{\mathrm{Q}}^{c}$. We then see that the actual v.e.v. value, $v$, may not correspond to the critical value $\lambda_{\mathrm{Q}}^{c}$. This brings about the question of how scale invariance is actually broken in our gap equations, and whether there might be a dilaton [5-7, 45-49]. (When the $125 \mathrm{GeV}$ hint emerged from 2011 LHC data, [4547] already suggested that it might be a dilaton rather than the SM Higgs boson, while $[48,49]$ are from the (walking) technicolor perspective).

Rather than to approach this deeper problem, we try to obtain the decay constant $f_{G}$ of the Goldstone boson, which should be the same as the vacuum expectation value, $v$. Following the Pagels-Stokar formula [50] naively, we obtain

$$
f_{G}^{2}=\frac{N_{C}}{4 \pi^{2}} \int_{0}^{\Lambda^{2}} d x \frac{x B(x)^{2}-(1 / 4) x\left(B(x)^{2}\right)^{\prime}}{\left(x A(x)^{2}+B(x)^{2}\right)^{2}} .
$$


More generally, we write

$$
\begin{aligned}
f_{G}^{2} & =\int_{0}^{\Lambda^{2}} d x \mathscr{F}[A(x), B(x)] \\
& =m_{\mathrm{Q}}^{2} \int_{0}^{\Lambda^{2} / m_{\mathrm{Q}}^{2}} d \widehat{x} \widehat{\mathscr{F}}\left[A(\widehat{x}), B(\widehat{x}) / A(\widehat{x}) / m_{\mathrm{Q}}\right],
\end{aligned}
$$

where $m_{\mathrm{Q}} \equiv B(0) / A(0)$, and we have scaled by $m_{\mathrm{Q}}$ (and redefined the function $\mathscr{F})$, treating it as physical.

We can get back the "Yukawa" coupling $\lambda$ (it should really be denoted as $Y$, and the question is whether $Y=\lambda$ ) for the input to the gap equation, or

$$
\frac{1}{2 \lambda^{2}}=\int_{0}^{\Lambda^{2} / m_{\mathrm{Q}}^{2}} d \widehat{x} \widehat{\mathscr{F}}\left[A(\widehat{x}), B(\widehat{x}) / A(\widehat{x}) / m_{\mathrm{Q}}\right] .
$$

If the system is really scale-invariant, the r.h.s. of (52) is a function of $\Lambda / m_{Q}$ and $\lambda$. In order to satisfy the gap equation, $\lambda$ is obtained as a function of $\Lambda / m_{Q}$. Namely, it should not depend on $m_{Q}$ explicitly. Taking the cutoff $\Lambda=2 m_{Q}$, the equation becomes iterative for $\lambda$. Therefore, solving the gap equation, we obtain a prediction for the heavy quark mass. But technical issues remain. Is $m_{\mathrm{Q}}$ a physical mass? What about the infrared cutoff? Can our assumption of $\lambda\left(\Lambda_{\mathrm{UV}} / m_{\mathrm{Q}}\right)=Y\left(\Lambda_{\mathrm{UV}} / m_{\mathrm{Q}}\right)$ be maintained self-consistently? Our work is far from complete, and we leave these theoretical questions to the future.

\section{New Phenomena: $Q \bar{Q} \rightarrow n V_{L}$}

The stringent limit of $m_{\mathrm{Q}} \gtrsim 700 \mathrm{GeV} / \mathrm{c}^{2}$ [14-20] is already above the perturbative, tree-level partial wave unitarity bound (UB) that is nominally around $550 \mathrm{GeV} / \mathrm{c}^{2}$ [21]. With $\mathrm{TeV}$ scale heavy quark masses, the actual UB violation (UBV) in the high energy limit for $Q \bar{Q}$ scattering may be out of reach. Instead, the question to ask is should the current search strategy for ultraheavy quark $Q$ at the LHC be modified? Of course, with the advent of the $125 \mathrm{GeV}$ boson at the LHC, the search for the four generation quarks has been blunted, and the search effort is being reshuffled to search for vector-like quarks, where flavor changing neutral current (FCNC) decays to $Z$ and Higgs bosons need to be studied. We maintain, however, that SM has built-in absence of FCNC, both for the $Z$ (only chiral fermions) and Higgs (single Higgs doublet). There is some merit to stick to this, while our bootstrap equation has even disposed of the Higgs doublet altogether.

So, what if there are heavy chiral quarks in Nature at the 2-3 TeV level? Can they be discovered at the LHC? We draw on the analogy with the proton to argue [52] that $Q \bar{Q} \rightarrow n G$ ( $G \equiv V_{L}$ is the longitudinal component of vector boson) may be the new signature at the LHC.

The $\pi N N$ coupling $g_{\pi N N}^{2} / 4 \pi \simeq 14[53,54]$ gives $g_{\pi N N} \simeq$ 13 , which is very large and quite close to the $\pi N N$ "Yukawa coupling," $\lambda_{\pi N N} \equiv \sqrt{2} m_{N} / f_{\pi} \simeq 14$, which is slightly larger than $4 \pi$. Although $\lambda_{\mathrm{Q}} \equiv \sqrt{2} m_{\mathrm{Q}} / v \gtrsim 3.5(v \cong 246 \mathrm{GeV}$ is the electroweak symmetry breaking scale) from the current
$m_{Q}$ bound is not yet as large, drawing analogy with $p \bar{p}$ annihilation, we expect that $Q \bar{Q} \rightarrow n G$ may be the dominant process for $m_{Q} \in(1,2) \mathrm{GeV}$. It is also realizing the fact that $\lambda_{\pi N N} \simeq g_{\pi N N}$ is of similar strength to $12 \lesssim \lambda_{Q}^{c} \lesssim 17$, that such strong couplings already exist in Nature, which gives some confidence to our very large critical Yukawa coupling strength.

4.1. Phenomenology of $p \bar{p} \rightarrow n \pi$. The phenomenon for $p \bar{p}$ annihilation is unfamiliar to the average particle physicist. One tends to think of annihilation into photons (much suppressed by $\alpha$ of QED) or gluons. But even for the latter, it is not practical with total energy of $\sim 1.88 \mathrm{GeV}$ and in consideration of detection of final states. Certainly, $p \bar{p}$ would annihilate into mesonic resonances. However, all mesonic resonances basically end up as pions. Thus, one can safely conclude that $p \bar{p} \rightarrow n \pi$ in general.

Indeed, it is observed that $p \bar{p}$ annihilation $[51,55]$ goes mainly into $n$ pions. However, the features are rather surprising, even for the nuclear and hadron physicists that worked on the subject from the 1950s to the 1990. It is found that $p \bar{p} \rightarrow n \pi$ goes via a "fireball." The salient features of the annihilation "fireball" are (see Figure 8)

(i) size of order $1 / m_{\pi}$;

(ii) temperature $T \simeq 120 \mathrm{MeV}$;

(iii) average number of emitted pions $\left\langle n_{\pi}\right\rangle \simeq 5$;

(iv) a soft-pion $p_{\pi}^{2} / E_{\pi}^{2}$ factor modulates the MaxwellBoltzman distribution for the pions.

It is worthwhile to elucidate these features a little further. The size $1 / m_{\pi}$ means that, when the $p$ and $\bar{p}$ meet to annihilate, destined to shed the $p$ and $\bar{p}$ content, the region of annihilation extends over a region $\sim 1 / m_{\pi}$. The system seems to thermalize to a temperature $T_{p \bar{p}}$ of order $120 \mathrm{MeV}$, hence, "loses memory" of its origins, and the emitted pions carry momenta that satisfy a thermal distribution. This rapid thermalization probably takes place due to the rather large $\pi N N$ (as well as $\pi \pi$ ) coupling, while the $p_{\pi}^{2} / E_{\pi}^{2}$ suppression [56] (satisfied rather well by data; see Figure 12 of [51]) for low pion momentum from the thermal distribution reflects nothing but the Goldstone nature of pion couplings. That is, the $\pi$ as Goldstone boson couples derivatively, hence, cannot get emitted at zero momentum. This seems to explain the enhancement factor of 1.3 for the mean kinetic energy $\left\langle K_{\pi}\right\rangle \equiv$ $\left\langle E_{\pi}\right\rangle-m_{\pi}$ beyond equipartition expectation of (3/2) $T_{p \bar{p}}$. Thus, the relatively high $\left\langle E_{\pi}\right\rangle \sim 370 \mathrm{MeV}$ gives rise to $\left\langle n_{\pi}\right\rangle \simeq$ 5.1 , as compared to the maximal allowed number of pions, $2 m_{N} / m_{\pi} \sim 13$.

At a more refined level, it is found that $\left\langle n_{\pi^{ \pm}}\right\rangle \simeq 3.1$ and $\left\langle n_{\pi^{0}}\right\rangle \simeq 2.1$, with $2\left\langle n_{\pi^{0}}\right\rangle /\left\langle n_{\pi^{ \pm}}\right\rangle>1$; that is, more neutral 
TABle 1: Sample multiplicity distributions: $P_{p \bar{p}}(n)$ is the observed distribution for $p \bar{p} \rightarrow n \pi[51]$, while $P_{\mathrm{QQ}_{1(2)}}(n)$ is the $Q \bar{Q} \rightarrow n G$ distribution for $m_{\mathrm{Q}}=1$ (2) $\mathrm{TeV}$ according to (58), where $G \equiv V_{L}$ is the electroweak Goldstone boson.

\begin{tabular}{|c|c|c|c|c|c|c|c|c|c|c|c|c|c|c|c|c|c|}
\hline \multirow{2}{*}{$P(n)$} & \multicolumn{17}{|c|}{$n$} \\
\hline & 2 & 3 & 4 & 5 & 6 & 7 & 8 & 9 & 10 & 11 & 12 & 13 & 14 & 15 & 16 & 17 & 18 \\
\hline$P_{p \bar{p}}$ & $0.4 \%$ & $8 \%$ & $18 \%$ & $46 \%$ & $22 \%$ & $6 \%$ & $0.3 \%$ & - & & & & & & & & & \\
\hline$P_{\mathrm{QQ}_{1}}$ & $0.1 \%$ & $1 \%$ & $6 \%$ & $19 \%$ & $31 \%$ & $27 \%$ & $12 \%$ & $3 \%$ & $0.4 \%$ & - & & & & & & & \\
\hline$P_{\mathrm{QQ}_{2}}$ & & & & & - & $0.2 \%$ & $0.9 \%$ & $3 \%$ & $8 \%$ & $16 \%$ & $22 \%$ & $22 \%$ & $16 \%$ & $8 \%$ & $3 \%$ & $0.9 \%$ & $0.2 \%$ \\
\hline
\end{tabular}
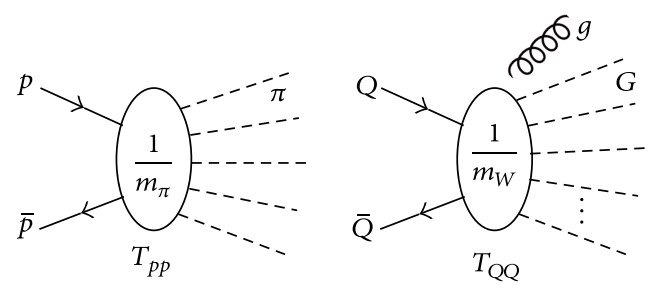

FIGURE 8: Illustration for $p \bar{p} \rightarrow n \pi$ for $n=\left\langle n_{\pi}\right\rangle \cong 5$ and the analog of $Q \bar{Q} \rightarrow n G$, where $G=V_{L}$ is the Goldstone boson of EWSB. Depending on $m_{\mathrm{Q}}=1-2 \mathrm{GeV},\left\langle n_{\mathrm{G}}\right\rangle$ could go from 6 to 12 (see (58) and Table 1). The gluon line indicates the relatively soft shedding of color.

pions are emitted than charged ones. Remarkably, the pion multiplicity distribution appears Gaussian,

$$
P\left(n_{\pi}\right)=\frac{1}{\sqrt{2 \pi} \sigma} e^{-\left(n_{\pi}-\left\langle n_{\pi}\right\rangle\right)^{2} / 2 \sigma^{2}},
$$

with $\sigma \sim 1$. More specifically [57],

$$
\sigma \simeq \frac{1}{2} \sqrt{\left\langle n_{\pi}\right\rangle}
$$

is argued from statistical models [58]. Thus, $\sigma \simeq 1.13$ gives a good fit to data [51], which is given in the first row of Table 1. Note the minute $p \bar{p} \rightarrow \pi \pi$ 2-body fraction, while the pion multiplicity cuts off above 8 .

This successful "statistical model" which accounts for gross features of $p \bar{p} \rightarrow n \pi$ annihilation goes back to Fermi [59], who considered a system of noninteracting pions. It has been refined through the years, and the strong interactions of the pions do play a major role. One final aspect is a focusing of incoming waves by attractive potential that leads to strong absorption in a smaller region than originally suggested.

4.2. $Q \bar{Q} \rightarrow n V_{L}$ Analog. We mean by $Q$ a left-handed chiral doublet (with corresponding right-handed weak singlets) that is degenerate in mass, thereby possessing a heavy isospin symmetry $I_{Q}$, much like the nucleon $N$. To draw true analogy with the $\pi N N$ case, the GQQ Yukawa coupling $\lambda_{\mathrm{Q}}$ should be of order 13-14; that is, $m_{\mathrm{Q}} \gtrsim 2 \mathrm{TeV}$, in accordance with findings of the bootstrap gap equation. However, for sake of phenomenology, we will assume that analogous phenomenon already appears for $1 \mathrm{TeV}$; hence, we consider $m_{\mathrm{Q}} \in(1,2) \mathrm{TeV}$.

With Higgs mechanism already established, the Goldstone boson $G \equiv V_{L}$ carries with it an effective length scale $1 / M_{W} \quad$ (assuming $I_{Q}$ symmetry, we take $M_{Z}=M_{W}$ and ignore weak gauge couplings $g, g^{\prime}$ compared with $\lambda_{\mathrm{Q}}$ ), which should define the size of the $Q \bar{Q}$ annihilation fireball. Compared with $m_{\pi} \propto m_{u}+m_{d}$, here $M_{W} \propto g$ where $g$ is the weak gauge coupling. Thus, the size of the fireball is controlled by random parameters such as $m_{q}$ or $g$ that are unrelated to the underlying annihilation dynamics.

The fireball temperature $T_{\mathrm{QQ}}$ is harder to assess. Noting that $T_{p \bar{p}} \sim 120 \mathrm{MeV}<T_{c}^{\mathrm{QCD}} \sim 170 \mathrm{MeV}$, likely $T<$ $T_{c}^{\mathrm{EW}}$, where $T_{c}^{\mathrm{EW}}$ is the electroweak transition temperature. By this analogy, however, one notes that $T_{c}^{\mathrm{QCD}}$ arises from the detailed underlying theory for hadron phenomena (which includes $p \bar{p} \rightarrow n \pi$ ), QCD. Even if EWSB arises from strong Yukawa coupling $[8,9], \lambda_{Q}$, we do not yet have an underlying theory for $\lambda_{Q}$ itself. Thus, we do not have a good handle on $T_{\mathrm{QQ}}$, except that it is in the $100 \mathrm{GeV}$ scale, of order $v$.

The Goldstone $p_{G}^{2} / E_{G}^{2}$ factor should still modulate the thermal $p_{G}$ distribution. But because of the smallness of $M_{W}^{2}$ compared with $4 m_{Q}^{2}$, the modulation is considerably milder than the $p \bar{p} \rightarrow n \pi$ case, and so $\left\langle K_{G}\right\rangle$ should be closer to $(3 / 2) T_{Q \bar{Q}}$. We shall therefore take as nominal

$$
T_{\mathrm{QQ}} \sim \frac{2}{3} v \sim 160 \mathrm{GeV}
$$

which can be interpreted as either $1.3 \times(1 / 2) v$ (here 1.3 corresponds to $\left.T_{p \bar{p}} / f_{\pi}\right)$ or $v \times T_{p \bar{p}} / T_{c}^{\mathrm{QCD}}$. The latter would give $170 \mathrm{GeV}$, which is not so different from (55). We stress, however, that the fireball temperature could be 1.5 , even twice as high, and should be determined eventually by experiment.

Assuming (55) but without applying the 1.3 enhancement factor over equipartition (as was done for the case of $p \bar{p} \rightarrow$ $n \pi)$, we take $\left\langle K_{G}\right\rangle \sim(3 / 2) T_{\mathrm{QQ}} \sim 240 \mathrm{GeV}$; hence, $\left\langle E_{\mathrm{G}}\right\rangle \sim$ $320 \mathrm{GeV}$, or

$$
\left\langle\left|p_{G}\right|\right\rangle \sim 310 \mathrm{GeV}
$$

with $\gamma_{G} \sim 4$. For $m_{\mathrm{Q}}=1(2) \mathrm{TeV}$, or $2 m_{\mathrm{Q}}=2(4) \mathrm{TeV}$, and this corresponds to

$$
\left\langle n_{G}\right\rangle \sim 6.25(12.5)
$$

where we artificially keep three digits of significance for generating a "realistic" multiplicity distribution. Assuming 
(53) and (54), we have $\sigma \simeq \sqrt{\left\langle n_{G}\right\rangle} / 2 \sim 1.25(1.77)$, and the multiplicity distribution is

$$
P\left(n_{G}\right) \simeq 0.319 e^{-\left(n_{G}-6.25\right)^{2} / 3.13}\left(0.226 e^{-\left(n_{G}-12.5\right)^{2} / 6.25}\right),
$$

for $m_{\mathrm{Q}}=1$ (2) TeV. We note that a higher fireball temperature $T_{Q \bar{Q}}$ would result in lower average multiplicity $\left\langle n_{G}\right\rangle$ and a narrower distribution (controlled by $\sigma$ ).

We illustrate the $Q \bar{Q} \rightarrow n G$ process in Figure 8 (gluon emission discussed later) and tabulate the multiplicity distributions in Table 1 . For $m_{Q}=1 \mathrm{TeV}$ (second row of Table 1 marked by $P_{\mathrm{QQ}_{1}}$ ), about $90 \%$ of $Q \bar{Q}$ annihilations go into 5-8 prongs of $V_{L} \equiv G$. Several $V_{L}$ s should be considerably above $300 \mathrm{GeV}$ momentum, while 4-prong events (at 6\%) are in general composed of $V_{L}$ s with momentum $\sim 500 \mathrm{GeV}$. Therefore, $W$-tagged "fat" jets, $j_{W}$, should become a useful tool for identifying these multi- $V_{L}$ events. For $m_{\mathrm{Q}}=2 \mathrm{TeV}$ (third row of Table 1 marked by $P_{\mathrm{QQ}_{2}}$ ), again over $90 \%$ of $Q \bar{Q}$ annihilations go into $10-15$ prong $V_{L} \mathrm{~s}$, which is a rather large number. For $9-12$ prong events (at $\sim 50 \%$ ), a significant number of $V_{L}$ s would have momentum above $400 \mathrm{GeV}$, while for higher multiplicity, many should still carry momentum higher than the mean, (56). These high multiplicity $n V_{L}$ events would be possible hallmark for heavy $Q \bar{Q}$ production.

4.3. LHC Prospects. At the moment, conventional wisdom has turned against searching for fourth generation quarks per se, because of the SM-like Higgs signal, even though a dilaton interpretation is still possible. Our discussion, however, point towards a possible new type of signature.

If our analogy with $p \bar{p}$ annihilation is already realized for $m_{\mathrm{Q}}=1 \mathrm{TeV}$, then even at $8 \mathrm{TeV}$ running of LHC, where of order $20 \mathrm{fb}^{-1}$ data is expected in 2012 for both ATLAS and CMS, one could already get a hint. The cross-section is of order a couple $\mathrm{fb}$; so, one might observe some number of 4 or more $W$-tagged jet $\left(j_{W}\right)$ events, with additional jet multiplicity that are less well $W$-tagged. The competing modes would be regular $Q \bar{Q}$ production, followed by "free quark decay," for example, (assuming $m_{b^{\prime}}>m_{t^{\prime}}$ ) $b^{\prime} \overline{b^{\prime}} \rightarrow$ $\bar{t} \bar{t} W^{+} W^{-} \rightarrow b \bar{b} W W W W$, or $t^{\prime} \bar{t}^{\prime} \rightarrow b \bar{b} W^{+} W^{-}$[60]; we shall assume CKM hierarchy for simplicity; hence, $b^{\prime} \rightarrow c, u$ and $t^{\prime} \rightarrow s, d$ transitions are ignored. We see that the $W$ jet multiplicity is lower, associated with isolated high $p_{T} b$ jets, and practically no $Z$-jets or $Z \rightarrow \ell^{+} \ell^{-}$. In contrast, $Q \bar{Q} \rightarrow n V_{L}$ does not have isolated $b$-jets ( $b$-jets would come in pairs at lower fraction, to form a $Z$ from $Z \rightarrow b \bar{b})$, and $W$ jet multiplicity is higher and tends to have $Z$-jets (the analogy with $p \bar{p} \rightarrow n \pi$ suggests a slight excess of $\pi^{0}$ over (half the) $\pi^{ \pm}$; however, the $Z$ mass is heavier than the $W$, and so this point is to be determined by experiment). We expect that the $Q \bar{Q} \rightarrow n V_{L}$ fireball process would dominate over the $Q \bar{Q} \rightarrow b \bar{b} W W(W W)$ free quark decay process, as we would argue shortly.

We have demonstrated in Section 3 that if the heavy chiral quarks $Q$ themselves are responsible [9] for dynamical EWSB, then $m_{\mathrm{Q}}>1 \mathrm{TeV}$ is required [8]. Our earlier analogy with the $\pi N N$ "Yukawa" coupling suggests $m_{\mathrm{Q}} \sim 2 \mathrm{TeV}$. If so, the prospects for the $2012 \mathrm{LHC}$ run at $8 \mathrm{TeV}$ are not good, and one would have to wait for the $13-14 \mathrm{TeV}$ run, expected by late 2014. Running the HATHOR code [61] for $Q \bar{Q}$ production, we estimate the $14 \mathrm{TeV}$ cross sections to be of order $50-60 \mathrm{fb}$ for $m_{\mathrm{Q}}=1 \mathrm{TeV}$, dropping to $\sim 3 \mathrm{fb}$ for $m_{\mathrm{Q}}=1.5 \mathrm{TeV}$, and $0.2-0.3$ $\mathrm{fb}$ for $m_{\mathrm{Q}}=2 \mathrm{TeV}$. From $2 m_{\mathrm{Q}}=2$ to $4 \mathrm{TeV}$, one quickly runs out of parton luminosity. Note that $q \bar{q} \rightarrow Q \bar{Q}$ production dominates over $g g \rightarrow Q \bar{Q}$ production, as the valence quark supplies the needed large parton momentum fraction.

From the cross section and expected LHC luminosities, for $m_{Q} \lesssim 1.5 \mathrm{TeV}$, again we do not foresee a problem for discovery. Note that assuming $I_{Q}$ symmetry, that is, near degeneracy of $t^{\prime}$ and $b^{\prime}$, then

(i) $t^{\prime}\left(b^{\prime}\right) \rightarrow b^{\prime}\left(t^{\prime}\right)+W^{*}$ decay: suppressed by both phase space and small Goldstone momentum;

(ii) $t^{\prime}\left(b^{\prime}\right) \rightarrow b(t)+W$ : suppressed by CKM element $\left|V_{t^{\prime} b}\right|$ $\left(\left|V_{t b^{\prime}}\right|\right)$, and with no sign of New Physics in $B_{s} \rightarrow$ $J / \psi \phi, B_{s} \rightarrow \mu^{+} \mu^{-}$, and $B_{d} \rightarrow K^{* 0} \mu^{+} \mu^{-}$, one expects $[62,63]$ such CKM elements to be less than 0.1.

In contrast, once $q \bar{q} \rightarrow Q \bar{Q}$ pulls the heavy quark pair out of the vacuum, the $Q \bar{Q}$ pair "sees" a cross section of order $1 / M_{W}^{2}$, which is at the $\mu b$ level. With $q \bar{q} \rightarrow Q \bar{Q}$ production, there is Yukawa attraction [24] between $Q \bar{Q}$ that mimics the focusing attraction for $p \bar{p} \rightarrow n \pi$. Thus, there is good likelihood that $Q \bar{Q} \rightarrow n G$, that is, $n V_{L}$, would dominate over free quark decay.

We comment that the produced $Q \bar{Q}$ is likely in a coloroctet state; hence, it would need to shed color. However, gluons have no way to sense the $T \sim 160 \mathrm{GeV}$ (or higher) of the fireball, which is of electroweak nature. Instead, the heaviness of $Q$ means gluon radiation is $1 / m_{Q}$ suppressed (heavy quark symmetry). We illustrate gluon radiation in Figure 8 but expect the associated gluon-jet to be soft and does not provide a discriminant.

In case $m_{Q} \gtrsim 1.5 \mathrm{TeV}$, one quickly runs out of parton luminosities (higher energy would be preferred!); hence, one would need high luminosity running of LHC at $14 \mathrm{TeV}$. However, the situation needs not be so pessimistic; the very large Yukawa coupling suggests the existence of bound states below $2 m_{\mathrm{Q}}$. For example, as discussed in [24], there is likely an isosinglet, color-octet $\omega_{8}$ resonance that can be produced via $q \bar{q} \rightarrow \omega_{8}$. How $\omega_{8}$ decays would depend on more details of the $Q \bar{Q}$ bound state spectrum and properties. The beauty of our analogy with $p \bar{p} \rightarrow n \pi$ annihilation is precisely the thermal nature of this fireball process $[51,55]$, with little "remembrance," either of the initial $p \bar{p}$ state or detailed resonances in the hadron spectrum. Thus, we make no assertion on $\omega_{8}$ decay properties here, except that it offers hope for an enhanced production cross section.

If the decay of the $\omega_{8}$ is analogous to the fireball picture, then by $m_{\omega_{8}}<2 m_{\mathrm{Q}}$ and the resonance production nature, there is good hope for earlier discovery. If $\omega_{8}$ decays through similar chains as discussed in [24], then it might lead to the discovery of several resonances. The study of [24] was 
done with $500 \mathrm{GeV}<m_{\mathrm{Q}}<700 \mathrm{GeV}$ in mind, to avoid issues of boundstate collapse $[9,23]$. But since this region is now close to being ruled out, a numerical update, in particular also on obtaining the spectrum, is certainly called for. This, however, requires nonperturbative solutions for strong Yukawa coupling.

An offshoot study of [24] provides an interesting contrast. If free quark decay is suppressed by very small $V_{t^{\prime} b}$, and some kinematic selections are operative, it is argued that $\omega_{8} \rightarrow \pi_{8}+W\left(\pi_{8}\right.$ is some isotriplet, color-octet Yukawabound "meson"), followed by $\pi_{8} \rightarrow W_{T}+g$, where $W_{T}$ is transverse. The upshot is the signature of $\omega_{8} \rightarrow W W g$. This is an exception to our fireball discussion, in that (1) it is effectively 2-body, $W W$, in vector bosons, and one of which is transverse; (2) the gluon is very energetic. If reconstructed [64], one could find two resonances simultaneously. These signatures arise from special conditions that are unlikely to hold in general. Furthermore, [24] did not consider fireball-like decays of $Q \bar{Q}$ annihilation by multi-Goldstone radiation.

Multiple weak boson production has been considered below $Q \bar{Q}$ threshold [65]. There is a rise of high multiplicities as one starts to approach threshold of high $m_{\mathrm{Q}}$. But that would be out of the range of validity for the $g g \rightarrow$ $n G$ amplitude via virtual $Q$ loop considered. We remark that our multi- $V_{L}$ signature is in principle quite distinct from micro-blackhole production [66]. Micro-blackholes in essence emit all types of particles democratically. In contrast, our fireball is heated in the electroweak sense and prefers emitting by far the strongly coupled weak Goldstone bosons $V_{L} \equiv G$. However, since searches so far are based only on the simplified signature of high jet multiplicities, a refined search is needed to separate micro-blackholes from $Q \bar{Q}$ fireballs.

We give a final remark on $V_{L} V_{L}$ scattering. If a very heavy chiral quark doublet $Q$ exists above the TeV scale, it would not be easily compatible with a light Higgs because of the very large quadratic corrections to the light Higgs mass, as we have already remarked. One would have to check whether the $125 \mathrm{GeV}$ boson is more consistent with a SM Higgs boson, or something like a dilaton. If the latter emerges, a corollary of our argument would then suggest that the traditional $V_{L} V_{L} \rightarrow V_{L} V_{L}$ scattering study for heavy or strongly coupled Higgs case may be the wrong place to search for New Physics enhancement. Instead, one should again watch out for $V_{L} V_{L}$ scattering to high(er) multiplicity of $V_{L}$ s. In general, one should treat the UBV in $V_{L} V_{L}$ and $Q \bar{Q}$ scattering as one single problem.

\section{Discussion and Conclusion}

Although the numbers arose from dynamical considerations-for sake of EWSB-rather than from constraints, from a phenomenological standpoint, our numerical value of $m_{\mathrm{Q}} \sim 3 \mathrm{TeV}$ in (49), even the $2 \mathrm{TeV}$ value in (48), seems depressingly high. At the same time, these masses are for the "no Higgs" case, or that the Higgs boson should be very heavy hence the contribution in the loop is subdominant, and we assert that the role of Higgs mechanism can be taken over dynamically by effective $Q \bar{Q}$ condensation. But a $125 \mathrm{GeV}$ boson has appeared at the LHC. Can a heavy chiral quark doublet still be viable? We offer few remarks, first on how $m_{\mathrm{Q}}$ might be lowered. We then address the $125 \mathrm{GeV}$ Higgs boson issue.

In the spirit of [9] and [24], the Goldstone boson $G$ is the lowest or most tightly bound $Q \bar{Q}$ state through the Yukawa coupling itself. There should exist Yukawa-bound resonances above this isotriplet, color-singlet, pseudoscalar state. The leading ones should be the isotriplet, pseudoscalar $\pi_{8}$ and the isosinglet, vector $\omega_{8}$, both of which are color-octet, and the isosinglet, color-singlet, vector $\omega_{1}$ "mesons." We have not yet solved the strongly coupled bound state problem; so, we do not yet know the actual spectrum (i.e., how tightly they are bound below $2 m_{\mathrm{Q}}$ ), nor the "decay constants," that is, how they couple to the heavy quark $Q$. But the couplings should be rather strong. The point is, as one integrates the Goldstone loop up to $2 m_{\mathrm{Q}}$, at some point these heavy mesons should also enter and contribute in the same spirit to the self-energy of $Q$.

There is thus some hope that extra, attractive contributions could lower $\lambda_{\mathrm{Q}}^{c}$, coming as additional effects of the large $\lambda_{\mathrm{Q}}$. But it also illustrates the limits of our bootstrap approach. The momentum integration for these extra contributions starts from the meson mass, up to $2 m_{Q}$, but clearly the meson propagators and the meson- $Q Q$ vertices would be much more sensitive to $q$, compared to the Goldstone boson $G$. Even for $G$, which is the $\pi_{1}$, as one approaches $2 m_{Q}$, its bound state nature would lead to modifications of its propagator (even if symmetry remains broken hence it remains massless) and vertex, which we have ignored in the ladder (or rainbow) approximation.

We have already offered our critique of the work of Hung and Xiong [25] and also showed numerically that the needed $\lambda_{\mathrm{Q}}^{c}$ becomes exorbitantly high if one retains the light Higgs scalar in the loop. A different question is whether our gap equation is actually equivalent to the NJL model. We have already commented that for the NJL model, the self-energy does not depend on momentum, and one simply cuts the loop momentum off at some $\Lambda$. The wave function part, $A\left(p^{2}\right)$, for our gap equation carries momentum dependence; that is, the Yukawa loop always modifies the $Z$ factor. We already saw this in scale-invariant QED. For NJL, the cutoff $\Lambda$ is traded, together with the associated dimension -2 coupling constant $G$, for the physical $f_{\pi}$ and $m_{N}$, although, depending on the cutoff, there is a critical coupling $G_{\text {crit }}$ (see (6)). For our case, one cannot take arbitrary values for the cutoff. Instead, we argued that, because the Goldstone boson would become unbounded at some scale, at least by $2 m_{\mathrm{Q}}$, the loop momentum has to be cut-off below this heuristic, finite value. Further similarities and differences are noted in [9].

A fundamental difference from NJL model may be the implicit postulation that the dimension zero Yukawa coupling of the Goldstone boson to be the experimentally verified one related to the left-handed vector gauge coupling $g$ of massive quarks. If the Goldstone boson $G$ is an ultratight $Q \bar{Q}$ bound state, it has turned the effective $Q \bar{Q}$ dimensionality to 1 . In this 
sense, our gap equation may resemble the gauged NJL model $[43,44]$, in which the dimensionality of the bound state tends to 1 near the critical gauge coupling.

Our approach is also conceptually different from those descended from the top condensation model $[67,68]$, in which the gauged NJL is applied to EWSB. The self-energy in the top condensation model incorporates both our Figure 4 (loop with four-quark operator) and Figure 5 (but with vector boson in the loop). The gap equation with the four-quark operator is equivalent to the minimization of the linear sigma model with a compositeness condition, such that the Yukawa and Higgs quartic couplings blow up at the composite scale. Naively speaking, our gap equation of Figure 6 corresponds to the linear sigma model with large Yukawa coupling. One can therefore read off the schematic correspondence between top condensation and our approach by replacing gauge coupling with Yukawa coupling, and four-quark operator loop by possible heavy bound state loop mentioned earlier. In the top condensation model, the four-quark interaction generates the large Yukawa coupling; hence, it is clearly rather different from our approach.

We were intrigued to find that the physically measured $g_{\pi N N}$ coupling (extracted via one-pion exchange in Born approximation) is consistent with $\lambda_{\pi N N} \equiv \sqrt{2} m_{N} / f_{\pi}$ and is rather similar in value to our finding in (46). This offers a totally separate argument that $m_{\mathrm{Q}}$ is in fact above $2 \mathrm{TeV}$. Having made this analogy of $\pi-N$ with $G-Q$, it is then interesting to ask whether the pion could really have been an $N \bar{N}$ bound state, as originally conjectured by Fermi and Yang [69], through our gap equation. However, developments in hadron physics subsequent to the Fermi-Yang conjecture relatively quickly gave rise to meson states in the 500 to $800 \mathrm{MeV}$ range (and corresponding baryon resonances), eventually exploding in the 1 to $2 \mathrm{GeV}$ range, that is, below $2 m_{N}$. Thus, our gap equation does not apply to the $\pi-N$ and hadron system case. Put simply, in such a $\pi-N$ gap equation, one cannot integrate the pion loop up to $2 m_{N}$; new phenomena emerged when the loop momentum $q$ reached $\sim \Lambda_{\mathrm{QCD}}$, which is quite below $2 m_{N}$. We know that the $\pi$ and $N$ are QCD bound states, with mesons formed by string breaking, and so the pion is not an ultratight bound state of $N$ and $\bar{N}$. If the $Q \bar{Q}$ ultratight bound state picture for the Goldstone $G$ could be realized according to our bootstrap gap equation, it would strengthen our reasoning [9] that the underlying theory for Yukawa couplings cannot be a simple mock-up of QCD.

We do not have new insight on bound state phenomena, other than what is already discussed in [24]. Unfortunately, this reference was very conservative and did not discuss above $m_{\mathrm{Q}}>700 \mathrm{GeV}$. The reason to keep this bound on $m_{\mathrm{Q}}$ is that, above this value, the Bethe-Salpeter (BS) equation approach tends to have collapsed states. But this was in turn the foundation for the postulate in [9], that the leading collapsed state, $\pi_{1}$, is precisely the Goldstone boson $G$, which in turn motivated the bootstrap gap equation study. We do not know at present whether our gap equation, with its numerical solution [8], could shed light on the $Q \bar{Q}$ bound state spectra, but the SD equation itself is "higher level" than the BS equation. If the BS equation Yukawa-boundstate approach can be a guide for $m_{\mathrm{Q}}$ as high as $2 \mathrm{TeV}$, that is, with $\alpha_{\mathrm{Q}}>1$, the noteworthy point is that the leading bound states $\omega_{1}, \omega_{8}$, and $\pi_{8}$ are rather distinct from the $\rho$ 's and $\eta$ 's of technicolor (TC), which after all is an extension of QCD. Note that these bound states emerge from strong Yukawa coupling, even though we have not offered a theory of Yukawa couplings (nor did we touch on the flavor aspect of Yukawa couplings). We mention in passing that our gap equation can be easily extended to finite temperature, allowing one to potentially explore issues related to electroweak phase transition, which is a direction that we would take up in a subsequent work.

We turn now to the difficult question of how to deal with the $125 \mathrm{GeV}$ boson that has emerged $[1,2]$ at the LHC. It is rather likely that this is the long-awaited SM Higgs boson. There are two issues against the existence of a 4 th generation chiral doublet if one has a $125 \mathrm{GeV}$ Higgs boson, as compared with having the Higgs boson above $600 \mathrm{GeV}$ or so (the original premise or working assumption in the formulation of the bootstrap gap equation). The first issue is the expected enhancement of $g g \rightarrow h$ production by roughly an order of magnitude due to 4 th generation quarks seen by the gluons in the loop [70]. But data indicate $[1,2]$ that $g g \rightarrow h \rightarrow V V$ seem not inconsistent with SM expectations. A second issue is if $m_{\mathrm{Q}} \gtrsim 700 \mathrm{GeV}$ [14-20], which is 4 times the top mass, what keeps the Higgs mass light? The top quark contribution to the Higgs mass quadratic correction is already a major concern. An effect from the fourth generation quarks that is an order of magnitude stronger seems rather difficult to tame. From a theoretical standpoint, the second problem is more serious.

It is possible to extend the Higgs sector, for example, to two Higgs doublets, where one could have one neutral Higgs boson light [71-73], and accommodate the Higgs data. Such phenomenological accommodation (where the Higgs fields could be composite), however, does not help our cause. Having two Higgs doublets introduces several more parameters, which we did not consider in the formulation of our gap equation. Furthermore, if these Higgs scalars couple with some modulated Yukawa coupling, our gap equations suggest that they raise the 4 th generation quark mass (but the pseudoscalars may help). Conversely, these models would still have to face how to keep a neutral Higgs scalar light in the presence of strong Yukawa couplings. Quintessentially, however, our bootstrap gap equation is against the concept of an elementary Higgs field. In any case, having more than one Higgs doublet invalidates our gap equation formulation and would bring us out of scope.

There has been discussion that a light Higgs boson could be a pseudo-Goldstone boson (PGH) from some underlying strong dynamics [74]. In these models, the PGH still has SMlike Higgs boson couplings. This would not suffice for our gap equation, since we have shown that the presence of a light Higgs, whether a PGH or not, would drive $m_{Q}$ too high for comfort. Such large $m_{Q}$ as implied by (47) would create its own "hierarchy" problem. We are therefore left with the dilaton option.

We find it remarkable, within the strong dynamical EWSB setting, the dilaton is a recurring issue. And, if not fortuitous, 
as mentioned in the Introduction, that the enhanced $\gamma \gamma$ mode for the Higgs-like signal at the LHC permits a dilaton interpretation [5-7]. In our case, our gap equation has scaleinvariance, which we have in fact used in (42) towards finding our numerical solution. Although there was no attempt at the UV theory, it seems that our bootstrap gap equation permits a connection with an underlying scale-invariant or conformal theory (the AdS-CFT or holographic link; see [6] and references therein). It should be noted that our goal was dynamical EWSB, or generating v.e.v., $v$. But since we tacitly used $\lambda_{Q}=\sqrt{2} m_{Q} / v$ throughout; $v$ generation is equivalent with $m_{\mathrm{Q}}$ generation, hence, breaking of the scaleinvariance of the gap equation. But since we did not attempt any theory of Yukawa couplings, $\lambda_{\mathrm{Q}}$, the actual source of scale-invariance violation is left for the theory of Yukawa couplings. It should be noted that if the $125 \mathrm{GeV}$ object is a dilaton, its couplings are modified by $v / f$, where $f$ is the dilaton decay constant (hence, VBF and VH production processes would be suppressed). Taking the bound that $v^{2} / f^{2} \lesssim 1 / 20$ (probably considerably smaller), the dilaton contribution in our gap equation would be self-consistently subdominant.

In conclusion, the ever-increasing mass bound on heavy sequential chiral quark $Q$ stimulates the question whether strong Yukawa coupling itself can be the source of electroweak symmetry breaking. A dynamical gap equation is argued, treating the Goldstone as massless inside the loop, coupling with chiral quarks $Q$ with the usual Yukawa couplings. By some analogy with scale-invariant QED, numerical solutions are found, such that dynamical EWSB is demonstrated. The resulting quark mass $m_{Q}$ seems to be in the $2-3 \mathrm{TeV}$ range. We favor the heavy or no Higgs scenario since $m_{\mathrm{Q}}$ would become exorbitantly high if a light Higgs boson is kept in the loop. Despite our rather high $m_{Q}$ value, LHC might still shed light on it. By analogy with the observed $p \bar{p} \rightarrow n \pi$ fireball annihilation, we conjecture that such very heavy chiral quarks that induce EWSB by their very large $(\gtrsim 4 \pi)$ Yukawa coupling may annihilate via $Q \bar{Q} \rightarrow n V_{L}$ with high multiplicity. Even with 2$3 \mathrm{TeV}$ quark mass, discovery may be aided by Yukawa-bound $Q \bar{Q}$ resonances such as the color-octet (heavy) isosinglet vector meson $\omega_{8}$, if it undergoes similar annihilation. With a $125 \mathrm{GeV}$ boson that has emerged at $\mathrm{LHC}$, to reconcile with a very heavy chiral quark doublet, that is, a 4 th generation, it must be a dilaton [5-7] of scale-invariance breaking.

\section{Acknowledgments}

The author thanks J. Alwall, J.-W. Chen, K.-F. Chen, H.-C. Cheng, T.-W. Chiu, T. Enkhbat, P. Q. Hung, K. Jensen, Y. Kikukawa, E. Klempt, H. Kohyama, T Kugo, C. N. Leung, C.-J. D. Lin, F. J. Llanes Estrada, M. Piai, and H. Yokoya for discussions and Y. Mimura and H. Kohyama for collaboration. This work is supported by NSC 100-2745-M-002-002ASP and various NTU grants under the MOE Excellence program.

\section{References}

[1] G. Aad, T. Abajyan, B. Abbott et al., "Observation of a new particle in the search for the standard model Higgs boson with the ATLAS detector at the LHC," Physics Letters B, vol. 716, no. 1, pp. 1-29, 2012.

[2] S. Chatrchyan, V. Khachatryan, A.M. Sirunyan et al., "Observation of a new boson at a mass of $125 \mathrm{GeV}$ with the CMS experiment at the LHC," Physics Letters B, vol. 716, no. 1, pp. 30-61, 2012.

[3] S. Chatrchyan, V. Khachatryan, A.M. Sirunyan et al., "Combined results of searches for the standard model Higgs boson in pp collisions at $\sqrt{s}=7 \mathrm{TeV}$," Physics Letters $B$, vol. 710, no. 1 , pp. 26-48, 2012.

[4] G. Aad, B. Abbott, J. Abdallah et al., "Combined search for the Standard Model Higgs boson using up to $4.9 \mathrm{fb}^{-1}$ of pp collision data at $\sqrt{s}=7 \mathrm{TeV}$ with the ATLAS detector at the LHC," Physics Letters B, vol. 710, no. 1, pp. 49-66, 2012.

[5] S. Matsuzaki and K. Yamawaki, "Is $125 \mathrm{GeV}$ techni-dilaton found at LHC?" Physics Letters B, vol. 719, no. 4-5, pp. 378-382, 2013.

[6] D. Elander and M. Piai, "The decay constant of the holographic techni-dilaton and the $125 \mathrm{GeV}$ boson," http://arxiv.org/abs/ 1208.0546 .

[7] Z. Chacko, R. Franceschini, and R. K. Mishra, "Resonance at $125 \mathrm{GeV}$ : Higgs or dilaton/radion?" http://arxiv.org/abs/ 1209.3259 .

[8] Y. Mimura, W. S. Hou, and H. Kohyama, "Bootstrap dynamical symmetry breaking with new heavy chiral quarks," http://arxiv.org/abs/1206.6063.

[9] W. S. Hou, "Some unfinished thoughts on strong Yukawa couplings," Chinese Journal of Physics, vol. 50, p. 375, 2012, http://arxiv.org/abs/1201.6029.

[10] B. Holdom, W. S. Hou, T. Hurth, M. L. Mangano, S. Sultansoy, and G. Ünel, "Four statements about the fourth generation," PMC Physics A, vol. 3, article 4, 2009.

[11] S. Chatrchyan, V. Khachatryan, A.M. Sirunyan et al., "Search for a Higgs boson decaying into a b-quark pair and produced in association with $b$ quarks in proton-proton collisions at $7 \mathrm{TeV}$," http://arxiv.org/abs/1302.2892.

[12] W. S. Hou, "Source of CP violation for Baryon asymmetry of the universe," Chinese Journal of Physics, vol. 47, p. 134, 2009, http://arxiv.org/abs/0803.1234.

[13] M. Kobayashi and T. Maskawa, "CP-violation in the renormalizable theory of weak interaction," Progress of Theoretical Physics, vol. 49 , no. 2, pp. 652-657, 1973.

[14] G. Aad, T. Abajyan, B. Abbott et al., "Search for pair production of heavy top-like quarks decaying to a high- $p_{\mathrm{T}} W$ boson and a $b$ quark in the lepton plus jets final state at $\sqrt{s}=7 \mathrm{TeV}$ with the ATLAS detector," Physics Letters B, vol. 718, no. 4-5, pp. 12841302, 2013.

[15] ATLAS Conference, ATLASCONF-2012-130.

[16] S. Chatrchyan, V. Khachatryan, A. M. Sirunyan et al., "Search for pair produced fourth-generation up-type quarks in $\mathrm{pp}$ collisions at $\sqrt{s}=7 \mathrm{TeV}$ with a lepton in the final state," Physics Letters B, vol. 718, no. 2, pp. 307-328, 2012.

[17] S. Chatrchyan, V. Khachatryan, A. M. Sirunyan et al., "Search for heavy bottom-like quarks in 4.9 inverse femtobarns of pp collisions at $\sqrt{s}=7 \mathrm{TeV}$," Journal of High Energy Physics, vol. 2012, article 123, 2012. 
[18] S. Chatrchyan, V. Khachatryan, A. M. Sirunyan et al., "Combined search for the quarks of a sequential fourth generation," Physical Review D, vol. 86, no. 11, Article ID 112003, 20 pages, 2012.

[19] S. Chatrchyan, V. Khachatryan, A. M. Sirunyan et al., "Search for heavy quarks decaying into a top quark and a $\mathrm{W}$ or $\mathrm{Z}$ boson using lepton + jets events in pp collisions at $\sqrt{s}=7 \mathrm{TeV}$,' Journal of High Energy Physics, vol. 2013, article 154, 2013.

[20] "Search for a heavy partner of the top quark with charge 5/3," CMS-PAS-B2G-12-003.

[21] M. S. Chanowitz, M. A. Furman, and I. Hinchliffe, "Weak interactions of ultra heavy fermions," Physics Letters B, vol. 78, no. 2-3, pp. 285-289, 1978.

[22] B. Holdom, "The discovery of the fourth family at the LHC: what if?" Journal of High Energy Physics, vol. 2006, article 76, 2006.

[23] P. Jain, D. W. McKay, A. J. Sommerer, J. R. Spence, J. P. Vary, and B. L. Young, "Isospin multiplet structure in ultraheavy fermion bound states," Physical Review D, vol. 49, no. 5, pp. 2514-2524, 1994.

[24] T. Enkhbat, W. S. Hou, and H. Yokoya, "Early LHC phenomenology of Yukawa-bound heavy $Q \bar{Q}$ mesons," Physical Review D, vol. 84, no. 9, Article ID 094013, 14 pages, 2011.

[25] P. Q. Hung and C. Xiong, "Dynamical electroweak symmetry breaking with a heavy fourth generation," Nuclear Physics B, vol. 848, no. 2, pp. 288-302, 2011.

[26] W. S. Hou and R. G. Stuart, "possibility of discovering the next charge $-1 / 3$ quark through its flavor-changing neutral-current decays," Physical Review Letters, vol. 62, no. 6, pp. 617-620, 1989.

[27] W. S. Hou and R. G. Stuart, "Flavor changing neutral currents involving heavy fermions: a general survey," Nuclear Physics B, vol. 320, no. 2, pp. 277-309, 1989.

[28] J. Beringer, J. F. Arguin, R. M. Barnett et al., "Review of particle physics," Physical Review D, vol. 86, no. 1, Article ID 010001, 1528 pages, 2012.

[29] G. 't Hooft and M. J. G. Veltman, "Regularization and renormalization of gauge fields," Nuclear Physics B, vol. 44, no. 1, pp. 189-213, 1972.

[30] G. 't Hooft, "Renormalizable Lagrangians for massive YangMills fields," Nuclear Physics B, vol. 35, no. 1, pp. 167-188, 1971.

[31] G. W. S. Hou, "A brief (p)review on a possible fourth generation world to come," in Proceedings of the 35th International Conference of High Energy Physics (PoS ICHEP' 10), vol. 244, Paris France, July 2010, http://pos.sissa.it//archive/ conferences/120/244/ICHEP\%202010_244.pdf.

[32] G. D. Kribs, T. Plehn, M. Spannowsky, and T. M. P. Tait, "Four generations and Higgs physics," Physical Review D, vol. 76, no. 7, Article ID 075016, 11 pages, 2007.

[33] K. Ishiwata and M. B. Wise, "Fourth generation bound states," Physical Review D, vol. 83, no. 7, Article ID 074015, 8 pages, 2011.

[34] T. Kugo, "Dynamical instability of the vacuum in the lagrangian formalism of the Bethe-Salpeter bound states," Physics Letters B, vol. 76, no. 5, pp. 625-630, 1978.

[35] P. Q. Hung and C. Xiong, "Implication of a quasi fixed point with a heavy fourth generation: the emergence of a TeV-scale physical cutoff," Physics Letters B, vol. 694, no. 4-5, pp. 430-134, 2011.

[36] P. Q. Hung and C. Xiong, "Renormalization group fixed point with a fourth generation: Higgs-induced bound states and condensates," Nuclear Physics B, vol. 847, no. 1, pp. 160-178, 2011.
[37] Y. Nambu and G. Jona-Lasinio, "Dynamical model of elementary particles based on an analogy with superconductivity. I," Physical Review, vol. 122, no. 1, pp. 345-358, 1961.

[38] Y. Nambu, "Quasi-particles and Gauge invariance in the theory of superconductivity," Physical Review, vol. 117, no. 3, pp. 648663, 1960.

[39] J. Goldstone, "Field theories with «superconductor» solutions," Il Nuovo Cimento, vol. 19, no. 1, pp. 154-164, 1961.

[40] J. Goldstone, A. Salam, and S. Weinberg, "Broken symmetries," Physical Review, vol. 127, no. 3, pp. 965-970, 1962.

[41] R. Fukuda and T. Kugo, "Schwinger-Dyson equation for massless vector theory and the absence of a fermion pole," Nuclear Physics B, vol. 117, no. 1, pp. 250-264, 1976.

[42] V. A. Miransky, "Dynamics of spontaneous chiral symmetry breaking and the continuum limit in quantum electrodynamics," Il Nuovo Cimento A Series 11, vol. 90, no. 2, pp. 149-170, 1985.

[43] W. A. Bardeen, C. N. Leung, and S. T. Love, "Dilaton and chiralsymmetry breaking," Physical Review Letters, vol. 56, no. 12, pp. 1230-1233, 1986.

[44] K. I. Kondo, H. Mino, and K. Yamawaki, "Critical line and dilaton in scale-invariant QED," Physical Review D, vol. 39, no. 8, pp. 2430-2433, 1989.

[45] V. Barger, M. Ishida, and W. Y. Keung, "Differentiating the Higgs Boson from the dilaton and radion at hadron colliders," Physical Review Letters, vol. 108, no. 10, Article ID 101802, 5 pages, 2012.

[46] V. Barger, M. Ishida, and W. Y. Keung, "Dilaton at the LHC," Physical Review D, vol. 85, no. 1, Article ID 015024, 4 pages, 2012.

[47] B. Coleppa, T. Gregoire, and H. E. Logan, "Dilaton constraints and LHC prospects," Physical Review D, vol. 85, no. 5, Article ID 055001, 12 pages, 2012.

[48] S. Matsuzaki and K. Yamawaki, "Techni-dilaton signatures at LHC," Progress of Theoretical Physics, vol. 127, no. 2, pp. 209-228, 2012.

[49] S. Matsuzaki and K. Yamawaki, "Techni-dilaton at $125 \mathrm{GeV}$," Physical Review D, vol. 85, no. 9, Article ID 095020, 5 pages, 2012.

[50] H. Pagels and S. Stokar, "Pion decay constant, electromagnetic form factor, and quark electromagnetic self-energy in quantum chromodynamics," Physical Review D, vol. 20, no. 11, pp. 29472952, 1979.

[51] E. Klempt, C. Batty, and J. M. Richard, "The antinucleonnucleon interaction at low energy: annihilation dynamics," Physics Reports, vol. 413, no. 4-5, pp. 197-317, 2005.

[52] W. S. Hou, "Searching for new heavy chiral quark pairs via their annihilation to multiple vector bosons," Physical Review D, vol. 86, no. 3, Article ID 037701, 5 pages, 2012.

[53] M. M. Pavan, R. A. Arndt, I. I. Strakovsky, and R. L. Workman, "Determination of the $\pi$ NN coupling constant in the VPI/GWU $\pi \mathrm{N} \rightarrow \pi \mathrm{N}$ partial-wave and dispersion relation analysis," Physica Scripta, vol. 2000, article 65, 2000.

[54] T. E. O. Ericson, B. Loiseau, and A. W. Thomas, "Determination of the pion-nucleon coupling constant and scattering lengths," Physical Review C, vol. 66, no. 1, Article ID 014005, 19 pages, 2002.

[55] C. B. Dover, T. Gutsche, M. Maruyama, and A. Faessler, "The physics of nucleon-antinucleon annihilation," Progress in Particle and Nuclear Physics, vol. 29, pp. 87-173, 1992.

[56] S. J. Orfanidis and V. Rittenberg, "Soft-pion limits of the inclusive pion distributions in $\bar{N} \mathrm{~N} \rightarrow \pi^{ \pm}+$anything at rest," Nuclear Physics B, vol. 56, no. 2, pp. 561-564, 1973. 
[57] S. J. Orfanidis and V. Rittenberg, "Nucleon-antinucleon annihilation into pions," Nuclear Physics B, vol. 59, no. 2, pp. 570-582, 1973.

[58] A. Jabs, "Lorentz-invariant and non-invariant momentum space and thermodynamics," Nuclear Physics B, vol. 34, no. 1, pp. 177-188, 1971.

[59] E. Fermi, "High energy nuclear events," Progress of Theoretical Physics, vol. 5, no. 4, pp. 570-583, 1950.

[60] A. Arhrib and W. S. Hou, "Flavor changing neutral currents involving heavy quarks with four generations," Journal of High Energy Physics, vol. 2006, article 9, 2006.

[61] M. Aliev, H. Lacker, U. Langenfeld, S. Moch, P. Uwer, and M. Wiedermann, "HATHOR-HAdronic Top and Heavy quarks crOss section calculatoR," Computer Physics Communications, vol. 182, no. 4, pp. 1034-1046, 2011.

[62] W. S. Hou, M. Kohda, and F. Xu, "Measuring the fourthgeneration $b \rightarrow s$ quadrangle at the LHC," Physical Review D, vol. 84, no. 9, Article ID 094027, 7 pages, 2011.

[63] W. S. Hou, M. Kohda, and F. Xu, "Hints for a low $B_{s} \rightarrow \mu^{+} \mu^{-}$ rate and the fourth generation," Physical Review D, vol. 85, no. 9, Article ID 097502, 5 pages, 2012.

[64] J. Alwall, T. Enkhbat, W. S. Hou, and H. Yokoya, "Doubly resonant $W W$ plus jet signatures at the LHC," Physical Review D, vol. 86, no. 7, Article ID 074029, 8 pages, 2012.

[65] K. Hagiwara and H. Murayama, "Multiple weak-boson production via gluon fusion," Physical Review D, vol. 41, no. 3, pp. 10011004, 1990.

[66] S. Chatrchyan, V. Khachatryan, A. M. Sirunyan et al., "Search for microscopic black holes in pp collisions at $\sqrt{s}=7 \mathrm{TeV}$," Journal of High Energy Physics, vol. 2012, article 61, 2012.

[67] V. A. Miransky, M. Tanabashi, and K. Yamawaki, "Dynamical electroweak symmetry breaking with large anomalous dimension and t quark condensate," Physics Letters B, vol. 221, no. 2, pp. 177-183, 1989.

[68] W. A. Bardeen, C. T. Hill, and M. Lindner, "Minimal dynamical symmetry breaking of the standard model," Physical Review D, vol. 41, no. 5, pp. 1647-1660, 1990.

[69] E. Fermi and C. N. Yang, "Are mesons elementary particles?" Physical Review, vol. 76, no. 12, pp. 1739-1743, 1949.

[70] A. Djouadi and A. Lenz, "Sealing the fate of a fourth generation of fermions," Physics Letters B, vol. 715, no. 4-5, pp. 310-314, 2012.

[71] S. Bar-Shalom, S. Nandi, and A. Soni, “Two Higgs doublets with fourth-generation fermions: models for TeV-scale compositeness," Physical Review D, vol. 84, no. 5, Article ID 053009, 24 pages, 2011.

[72] X. G. He and G. Valencia, "An extended scalar sector to address the tension between a fourth generation and Higgs searches at the LHC," Physics Letters B, vol. 707, no. 3-4, pp. 381-384, 2012.

[73] S. Bar-Shalom, M. Geller, S. Nandi, and A. Soni, "Two Higgs doublets, a 4th generation and a $125 \mathrm{GeV}$ Higgs: a review," http://arxiv.org/abs/1208.3195.

[74] G. F. Giudice, C. Grojean, A. Pomarol, and R. Rattazzi, "The strongly-interacting light Higgs," Journal of High Energy Physics, vol. 2007, article 45, 2007. 

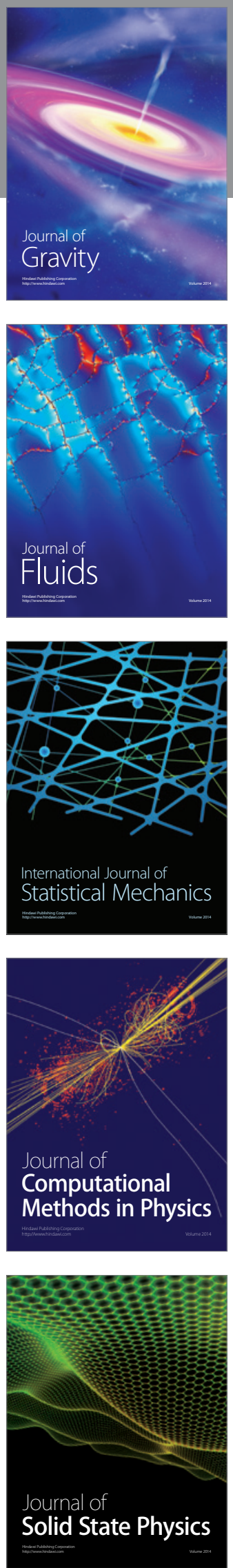

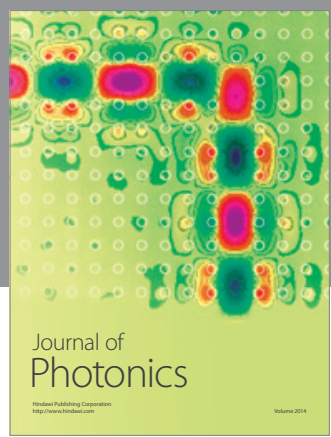

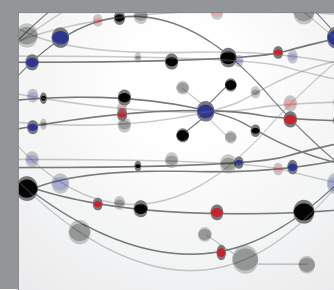

The Scientific World Journal

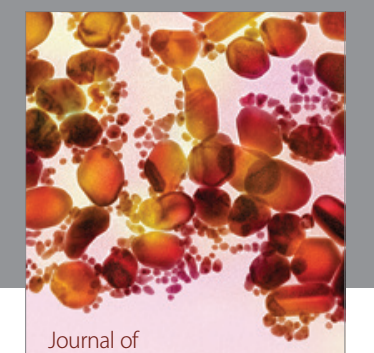

Soft Matter
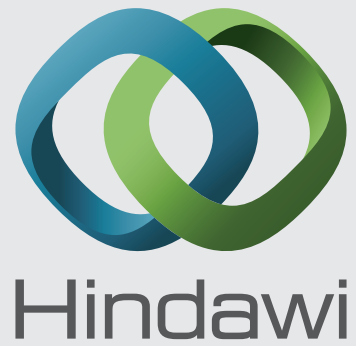

Submit your manuscripts at

http://www.hindawi.com
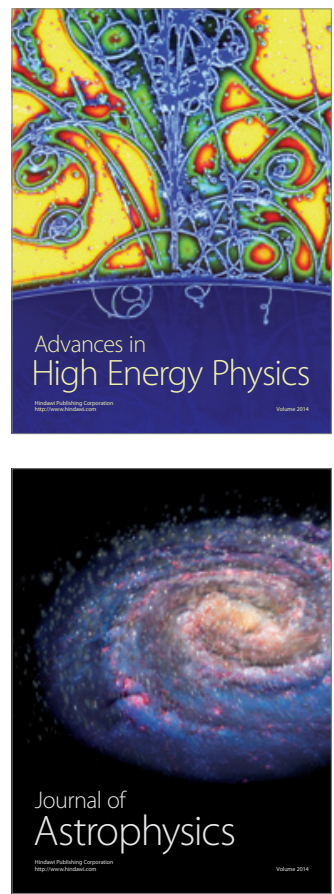
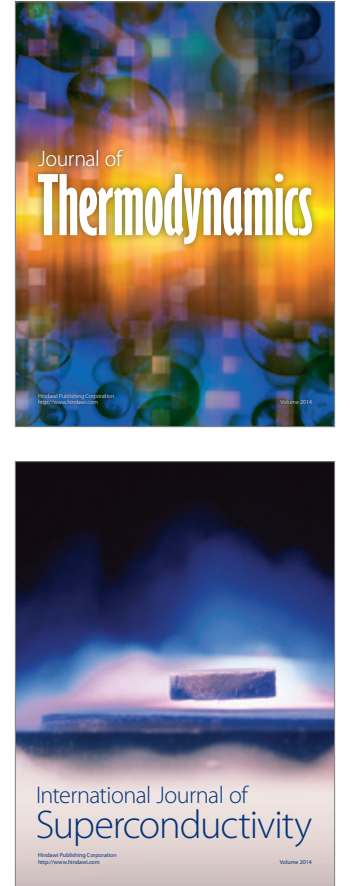
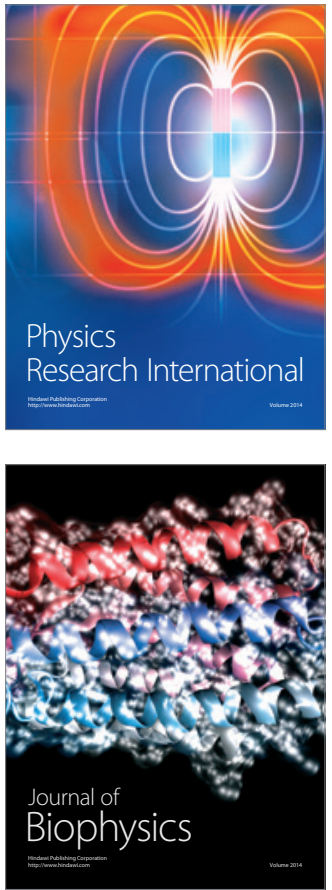
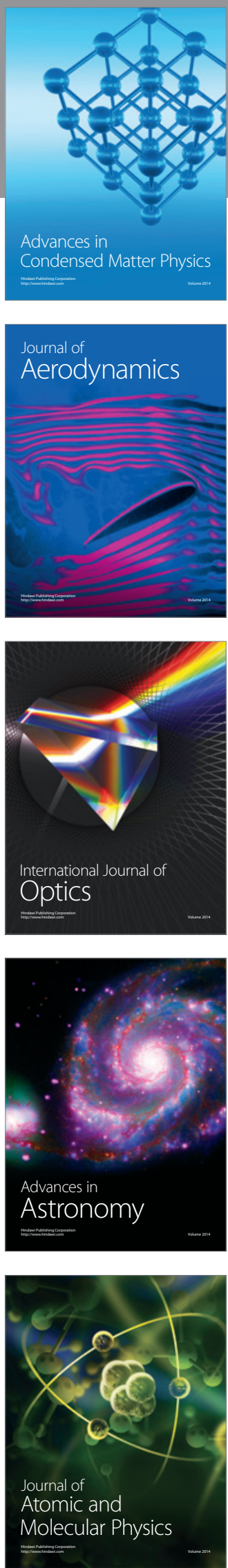